\title{
Pneumatic Long-Wave Generation of Tsunami-Length Waveforms and their Runup
}

\author{
D. J. McGovern ${ }^{\mathrm{a}, *}$, T. Robinson ${ }^{\mathrm{b}}$, I. D. Chandler ${ }^{\mathrm{c}}$, W. Allsop ${ }^{\mathrm{c}}$, T. Rossetto ${ }^{\mathrm{b}}$ \\ ${ }^{a}$ School of the Built Environment and Architecture, London South Bank University, 103 Borough Road, \\ London, SE1 OAA \\ ${ }^{b}$ Department of Civil, Environmental and Geomatic Engineering, University College London, London, UK, \\ WC1E $6 B T$ \\ ${ }^{c}$ HR Wallingford, Howbery Park, OX10 8BA, UK
}

\begin{abstract}
An experimental study is conducted using a pneumatic long-wave generator (also known as a Tsunami Generator). Scaled tsunami waveforms are produced with periods in the range of 5 to 230 seconds and wave amplitudes between 0.03 to 0.14 metres in water depths of 0.7 to 1.0 metres. Using Froude similitude in scaling, at scale 1:50, these laboratory waves are theoretically dynamically equivalent to prototype tsunami waveforms with periods between 1 to 27 minutes and positive wave amplitude between 1.5 to 7.0 metres in water depths of $50 \mathrm{~m}$. The purpose of these tests is to demonstrate that the pneumatic method can generate long waves in relatively short flumes and to investigate their runup. Standard wave parameters, (free-surface, wave celerity and velocity profiles) are used to characterise the waveforms. It is shown that for the purpose of runup and onshore ingression, minimal interference from the re-reflected waves is observed.

By generating tsunami waveforms with periods greater than $\approx 80 \mathrm{~s}(\approx 9.5$ mins prototype scale) the available experimental data set is expanded and used to develop a new runup equation. Contrary to the shorter waves, shoaling of these longer waves is insignificant. For waveforms with periods greater $\approx 100 \mathrm{~s}$ the runup is best described by wave steepness not potential energy. When tested against available runup equations the results are mixed; most perform poorly for scaled tsunami length periods. A segmented regression analysis is performed on the data set and an empirical runup relationship is provided based on a new parameter termed the 'Relative Slope Length'.
\end{abstract}

\footnotetext{
*Corresponding author

Email address: david.j.mcgovern1@gmail.com (D. J. McGovern)
} 
The tests show the definition of offshore wave amplitude is non-trivial and may greatly affect the predicted relative runup of a given wave. It is noted that this appears to be a general issue for all types of tsunami simulation in the laboratory. Together these observations and proposed runup model provide a framework for future numerical studies of the topic. Keywords: Experiments, Pneumatic Generation, Long Wave, Tsunami, Runup

\section{Introduction}

Tsunami waves are progressive gravity waves most commonly generated by under-sea mega-thrust fault motion. Their periods range between $\approx 90$ to $7000 \mathrm{~s}(\approx 1.5$ mins to 2 hrs, see Brown 2013) and they have sufficient potential energy to present a significant threat to coastal life and the built environment. The Indian Ocean tsunami in 2004 resulted in over two hundred and fifty thousand dead or missing, $\$ 9.9$ billion in material damage losses and 1.7 million displaced persons (Telford et al., 2006). Catalogues of past tsunami events are available (NOAA 2017a, NOAA 2017b and Geist and Parsons 2011) and demonstrate the destructive potential of tsunami waves. One of largest recent tsunami is the 2011 Japan event, commonly known as the 2011 Tohoku earthquake and tsunami. The human death toll, according to The National Police Agency of Japan (NPA, 2016) exceeds fifteen thousand. The economic impact measured over the succeeding year from the event is shown by Kajitani et al. (2013) to be over of 211 billion USD in direct damage.

One way of reducing human and economic losses from future tsunami events is through improved understanding of the inundation of tsunami on an coastline. Such improvements may lead to better engineering guidelines for coastal infrastructure that are at risk of large tsunami events. These are the main motivations of the presented research.

\subsection{Characterisation of tsunami encroaching on land}

One characterisation of the interaction of a tsunami with a coastline is its runup. Runup is defined as the vertical height above static water level of the point of maximum inundation of the tsunami inland. It is a commonly used parameter to describe tsunami-like waveforms in the laboratory (for example Synolakis 1987, Tadepalli and Synolakis 1994, Briggs et al. 1995, Liu et al. 1995, Hughes 2004a, Madsen and Schäffer 2010, Charvet et al. 2013, Saelevik et al. 2013, Sriram et al. 2016 and Drähne et al. 2016), and in the assessment of tsunami interaction with a shoreline, particularly for risk analysis, planning and insurance (for example, Imamura 2009 and ASCE/SEI 2017). 
More recently, tsuanmi inundation of the coastline and their over-land flow are also characterised by parameters such as flow velocity and depth. The ASCE/SEI 2017 'Tsunami Loads and Effects' design standard outlines the energy grade line method to analyse the 2-dimensional tsunami flow inundation depth and velocity at a specified point onshore. Its use requires the maximum runup and inundation of a given wave and its offshore period and amplitude as inputs. Taubenböck et al. (2013) present the application of the specific energy head to assess the inundation of tsunami on a coastline incorporating the flow depth and velocities. These parametrisations are important when consideration of the tsunami overland flow and velocities is desired. However, relating runup to offshore tsunami parameters remains important to improving mitigative engineering and planning of coastlines. This paper focuses on runup as the parameter that describes tsunami interaction with a coastline.

Early laboratory work on tsunami runup is based on solitary wave theory (for example, Synolakis 1987, Briggs et al. 1995, Liu et al. 1995, Chang et al. 2009 and Saelevik et al. 2013). A solitary wave centred at $X_{1}$ and $t=0$ has a free surface profile described by

$$
\eta(X, 0)=\frac{H}{d} \operatorname{sech}^{2}\left(K_{s}\left(X-X_{1}\right)\right)
$$

where $H$ is wave height, $d$ is the water depth and $K_{s}=1 / d \sqrt{3 H / 4 d}$. However, the work by Madsen et al. (2008) shows that the distance over which an arbitrary waveform develops into a solitary wave is generally greater than the typical geophysical scales of the prototype. They conclude that the solitary wave is an inappropriate model analouge for a tsunami wave at prototype.

First proposed by Tadepalli and Synolakis (1994), tsunami are also modelled using the $N$ wave assumption, (E.g., Madsen and Schäffer 2010 and Sriram et al. 2016). When extended in duration this provides a more realistic representation of prototype tsunami waveforms by accounting for the leading trough of the wave, as well as its period T. Madsen and Schäffer (2010) pose theoretical trough-led $N$-wave forms as

$$
\eta(X, 0)=\alpha \frac{H}{d}\left(X-X_{2}\right) \operatorname{sech}^{2}\left(K_{s}\left(X-X_{1}\right)\right)
$$

where $\alpha$ is a constant, $X_{1}$ is the position the crest and $X_{2}$ is the horizontal position of the zero-crossing point in the wave profile. Madsen and Schäffer (2010) use Equation (2) to derive new runup equations.

In line with the development of knowledge of the waveform, over the last ten years there 
has been a drive to improve the generation techniques of tsunami waves in the laboratory. Both solitary and trough-led waveforms have been used to measure the performance of various novel tsunami simulation techniques. Goseberg et al. (2013) introduces a pump technique to generate tsunami in a closed-circuit flume. The technique uses a Proportional Integral Derivative (PID) controller to generate target waves and absorb reflections. Drähne et al. (2016) use this pump methodology to investigate 'long wave' runup on a beach. While no definition of 'long wave' is given, the waves tested include waves of tsunami length in period if a notional scale of 1:100 is used. In theory the period and wave amplitude limitations could be overcome by increasing the pump capacity and the reservoir volume. A disadvantage of the method relates to spurious short period waves that are observed superimposed on the target wave. Also termed as 'riding waves' these waves in some cases overtake the target long wave being generated and directly interfere with the maximum runup of the long wave (Drähne et al., 2016). Such spurious waves are reduced in Bremm et al. (2015) by (to the current authors' understanding), bypassing the active PID control of the wave signal in realtime and inputting the smoothed form of the target wave signal. It is not immediately clear how the smoothed signal is achieved, but it is presumed that the method is similar to the iterative calibration of the target wave signal which is described later in the present work.

Schimmels et al. (2016) explore the use of a piston-paddle wave maker, however, the experimental scale, depth and amplitude are limited due to the maximum stroke of the wave maker. They report that '...the absolutely correct reproduction of the 'Mercator time series' with a piston type wave maker seems really to be unfeasible as the required stroke, although it only increases linearly with scale, becomes too large for very small water depth.' The 'Mercator' 2004 Indian Ocean Tsunami free-surface elevation time series is given in Appendix A, along with selected time series from the 2011 Great Eastern Japan Earthquake and Tsunami. The methodology is developed by Fernández et al. (2014) who use a SelfCorrecting Method (SCM) to numerically optimize the control variable, before applying it to a paddle to generate tsunami-length waveforms at 1:100 scale. This methodology adapts the control signal iteratively in the frequency domain by adjusting wave phase and amplitude to achieve the target $\eta(X, t)$. The method incorporates the absorption of re-reflections within the corrected control variable (paddle motion), and removes spurious high frequency components. After two correction steps the resulting long waveform shows good agreement in overall target wave period, though there is still some deviation from the smoothness of the target waveform time-series. This is particularly observable for actual tsunami time- 
series. Additionally, the amplitudes generated in this facility are significantly limited by the maximum stroke meaning the correct scaling of $a^{+}$and $d$ requires an exceptionally large paddle stroke. Furthermore, the SCM requires that the target wave be described meaningfully in the frequency domain by a set of linear sine waves, which may not be the case for highly non-linear waves or solitary waves.

Between 2008 and 2015, collaboration between University College London and HR Wallingford, U.K. developed and improved the design of a Pneumatic Long-Wave Generator (PLWG). The first generation PLWG is described in Rossetto et al. (2011) who introduce the concept and apply it to flume with a propagation region of constant depth of $15.2 \mathrm{~m}$. Waves are generated in an open-loop process between the control variable (the PLWG water head) and the output wave time-series. That is, the control variable time series is pre-calibrated for each wave. Sine waves up to $200 \mathrm{~s}$ in period are produced with the purpose of observing the response of the PLWG-flume system and the ability of the PLWG to reproduce simple periodic signals. Crest-led and trough-led waveforms are also produced with a maximum period of $\approx 18 \mathrm{~s}$ in order to check the repeatability of the PLWG and record wave runup for comparison with past experiments. The authors do not discuss wave absorption, and suggest future research with the PLWG method ought to include a longer constant-depth region in the flume (i.e., a longer flume) in order to increase the wavelength of the waves that can be generated.

Using the 1st generation PLWG and flume as described in Rossetto et al. (2011), Charvet et al. (2013) record the runup of crest-led 'elevated' and trough-led $N$-waves. Elevated waves are waves of translation characterised by a single positive elevation above the mean water level. They are nominally similar to a solitary wave but do not conform to its mathematical description, Equation (1), being generally much longer in length and therefore less steep than a solitary of equivalent amplitude. Charvet et al. (2013) compare elevated wave runup with solitary wave data of equivalent amplitude from Synolakis (1987) and find that elevated waves give a higher runup, suggesting measures other than amplitude such as wave energy might be important in the runup process.

They also provide evidence that the runup of 'very long waves' (defined as model period $T>\sim 11 \mathrm{~s}$ ) is different to that of 'long waves' ( $T<\sim 11 \mathrm{~s}$ ) and present runup relationships for $N$ - and elevated waves. The terms 'very long' and 'long' as described by (Charvet et al., 2013), are defined as waves of $T / T_{b}>1$ and $T / T_{b}<1$ respectively, where $T_{b}$ (Equation 3 ) is the time it takes for a given wave to travel the length of the beach $l_{\text {beach }}$. For the vast majority 
of tests, however, wave period did not exceed $10 \mathrm{~s}$, with only 4 waves exceeding 10 mins at $1 / 50$ scale. The maximum period is 1214 s at $1 / 50$ for an $N$-wave. The study highlights the potential influence of wave period, shape and steepness on tsunami runup and the need for further study of tsunami-like waves to understand their inundation characteristics.

$$
T_{b}=\int_{0}^{l_{\text {beach }}} \frac{d X}{\sqrt{g d\left(1-\frac{X}{l_{\text {beach }}}\right)}}=\frac{2 l_{\text {beach }}}{\sqrt{g d}}
$$

where $g$ is acceleration due to gravity and $X$ is the horizontal coordinate (1a).

The next steps in the development of the PLWG method is to apply it to a longer flume to investigate tsunami-length wave generation, absorption and reflection as well as extend the runup data of Charvet et al. (2013) to periods of tsunami-length. To this end, the development and commissioning of a 2nd generation PLWG came in 2015. A summary of the two facilities is given in Table 1.

Table 1: Comparison of the 1st and 2nd Generation PLWG

\begin{tabular}{clllllllc}
\hline Type & $\begin{array}{l}\text { length } \\
\text { height } \\
\text { width }(\mathrm{m})\end{array}$ & Volume $\left(\mathrm{m}^{3}\right)$ & $\begin{array}{l}\text { Flume } \\
\text { length } \\
(\mathrm{m})\end{array}$ & $\begin{array}{l}\text { length } \\
\text { of sloping } \\
\text { bathymetry } \\
\text { lbathy }(\mathrm{m})\end{array}$ & $\begin{array}{l}\text { slope } \\
\text { angle }\end{array}$ & $\mathrm{d}(\mathrm{m})$ & $T_{\max }(\mathrm{s})$ & $a_{\max }^{+}(\mathrm{m})$ \\
\hline 1st & $\begin{array}{l}4.8 \times 1.8 \times 1.15 \\
2 \text { nd }\end{array}$ & 9.94 & 19 & 13.8 & 2.86 & $0.45-0.69$ & 18 & 0.12 \\
\hline
\end{tabular}

The 2nd generation PLWG, whose set-up and operation is described in $\S 2$, is able to reproduce both trough and crest led tsunami-length waves in a $100 \mathrm{~m}$ long flume. Additionally, it is successfully able to recreate the full 'Mercator' 2004 Indian Ocean tsunami profile at correct 1/50 scaled water depth (Allsop et al., 2014). This, to the authors' knowledge has not yet been reproduced at correct scaled water depth in other flumes. Its set up and commissioning is described in Chandler et al. (2016) respectively, and the development process between the 1st and 2nd generations is described in Allsop et al. (2014).

This paper presents the experimental results from the first testing programme to be carried out using the 2nd generation PLWG. The aims of this paper are (1), to demonstrate that it is possible to generate a Froude-scaled tsunami-length wave in a flume that is significantly shorter than the incident wavelength and (2), to explore the runup and behaviour of waves that are of tsunami length. Aim (1) is the natural progression of the PLWG from the work of Rossetto et al. (2011) and Charvet et al. (2013). It directly addresses the effects 
of the lack of absorption at the generator and the open-loop generation method, as well as build on those works by increasing the period tested to tsunami-lengths and providing data repeatability at this period. Aim (2) builds on the available published data sets of runup by going some way to addressing the apparent gap in runup data for tsunami-length waves.

The laboratory set-up and methodology is presented first. Next, the analysis of the scaled waveforms and reflections is described, and the significance of the experimental runup results is discussed. The performance of appropriate available runup equations is evaluated against the new data set. An analysis of the parameters influencing runup is then presented, from which a new empirical prediction formula is regressed. A discussion is then made as to the influence of how offshore amplitude is defined on the runup measurement. Finally, the conclusions to the study are presented along with the proposed future research needs.

\section{Laboratory Set-up and Experimental Programme}

The PLWG is installed at the far end of the $100 \mathrm{~m}$ long $1.8 \mathrm{~m}$ wide flume at HR Wallingford, U.K. The length of the flume over which the wave may propagate is $65.6 \mathrm{~m}$, significantly longer than the previous PLWG flume in Rossetto et al. (2011). The new PLWG and larger flume set-up allows increased water depth ranges between $\approx 0.4$ to $1.0 \mathrm{~m}$ for runup tests. This improves upon Rossetto et al. (2011) and Charvet et al. (2013) in which water depths range from 0.45 to a limit of $0.69 \mathrm{~m}$. At the opposite end of the flume a 1:20 sloping bathymetry and runup beach is installed. The PLWG is a $4 \mathrm{~m}$ long $3.5 \mathrm{~m}$ high and $1.8 \mathrm{~m}$ wide machined steel box with a chamfered opening $0.4 \mathrm{~m} \times 1.8 \mathrm{~m}$ at the base (total volume $21.6 \mathrm{~m}^{3}$, Figure 1). This increased volume with resepct to the first generation PLWG allows for larger wave amplitudes to be generated for a given wavelength. Due to its larger size and volume, two vacuum pumps (a Zepher RT-95330 and an RT-84086) are used to pump air out of the PLWG via two $150 \mathrm{~mm}$ diameter pipes located on top of the steel box. The internal PLWG air pressure is varied by changing the angle of a computer-controlled butterfly valve in another pipe. This valve varies the net pressure and hence, the head of water within the PLWG, which is the control variable of the system. The output variable is the spatial and time-dependent free-surface elevation $\eta(X, t)$, where $X$ is the horizontal coordinate and $t$ is time. A flow shaper is used to control the water flow exiting the PLWG. A rectilinear coordinate system is used with $X=0$ being at the leading tip of the flow shaper, $Z$ being the vertical coordinate ( 0 at the flume bed) and $Y$ being the lateral coordinate ( 0 at the flume centreline). 

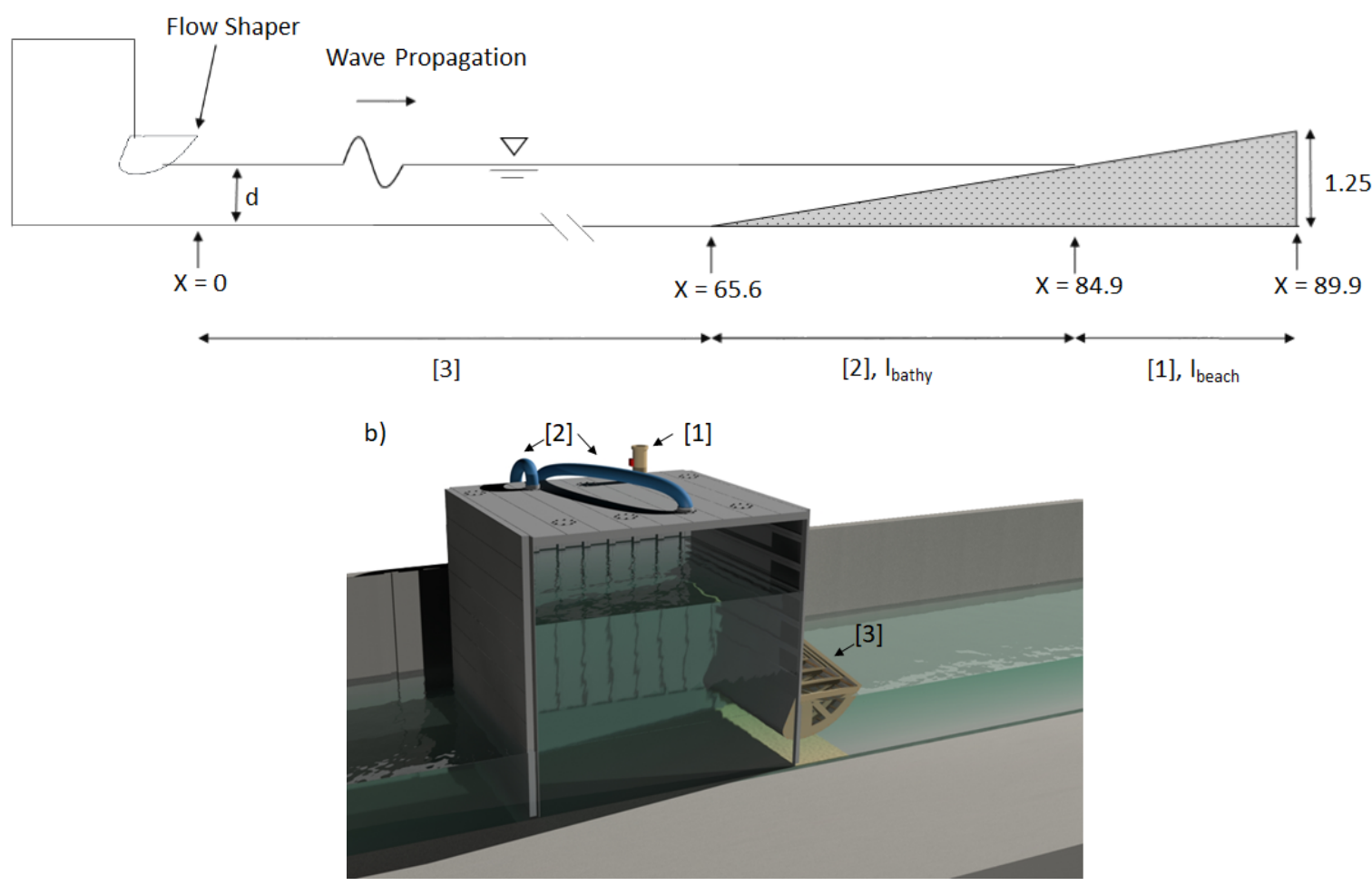

Figure 1: a) A schematic diagram of the flume. All distances are in metres (not to scale) with the onshore $\left(l_{\text {beach }}\right)$ [1], near-shore $\left(l_{\text {bathy }}\right)$ [2] and offshore (constant-depth) [3] regions shown. b) A computer rendered graphical representation of the PLWG showing the control valve [1], air pipes [2] and the flow shaper [3].

\subsection{Instrumentation and Data Collection}

The waveform $\eta(X, t)$, is recorded in the offshore (constant depth region $X=0$ to $65.6 \mathrm{~m}$ ), the nearshore (above the sloping bathymetry $X=65.6$ to $84.9 \mathrm{~m}$ ) and onshore (beach $X$ $=84.9$ to $89.9 \mathrm{~m}$ ) regions of the flume using 16 resistance-type wave gauges (accuracy \pm $0.0005 \mathrm{~m}$, manufactured by HR Wallingford). These consist of $0.9 \mathrm{~m}$ length gauges in the offshore and nearshore regions, and $0.3 \mathrm{~m}$ length in the onshore regions. These gauges are calibrated regularly and before each set of wave conditions. The calibration gradients, of which an $R^{2}$ of 0.9999 or better is demanded, are also recorded and compared throughout the experimental campaign to confirm consistency in the calibration fits and $R^{2}$ values across all calibrations. The runup is calculated by converting the maximum position the wave ingression up the beach slope to a vertical distance. A tape measure on the centreline of the beach allows the measurement to be made with an accuracy of $\pm 0.01 \mathrm{~m}$. All waves produced a relatively straight front indicating influence of the side wall and glass wall, both of which are very smooth, was limited. Comparisons of runup measured along the centreline and 


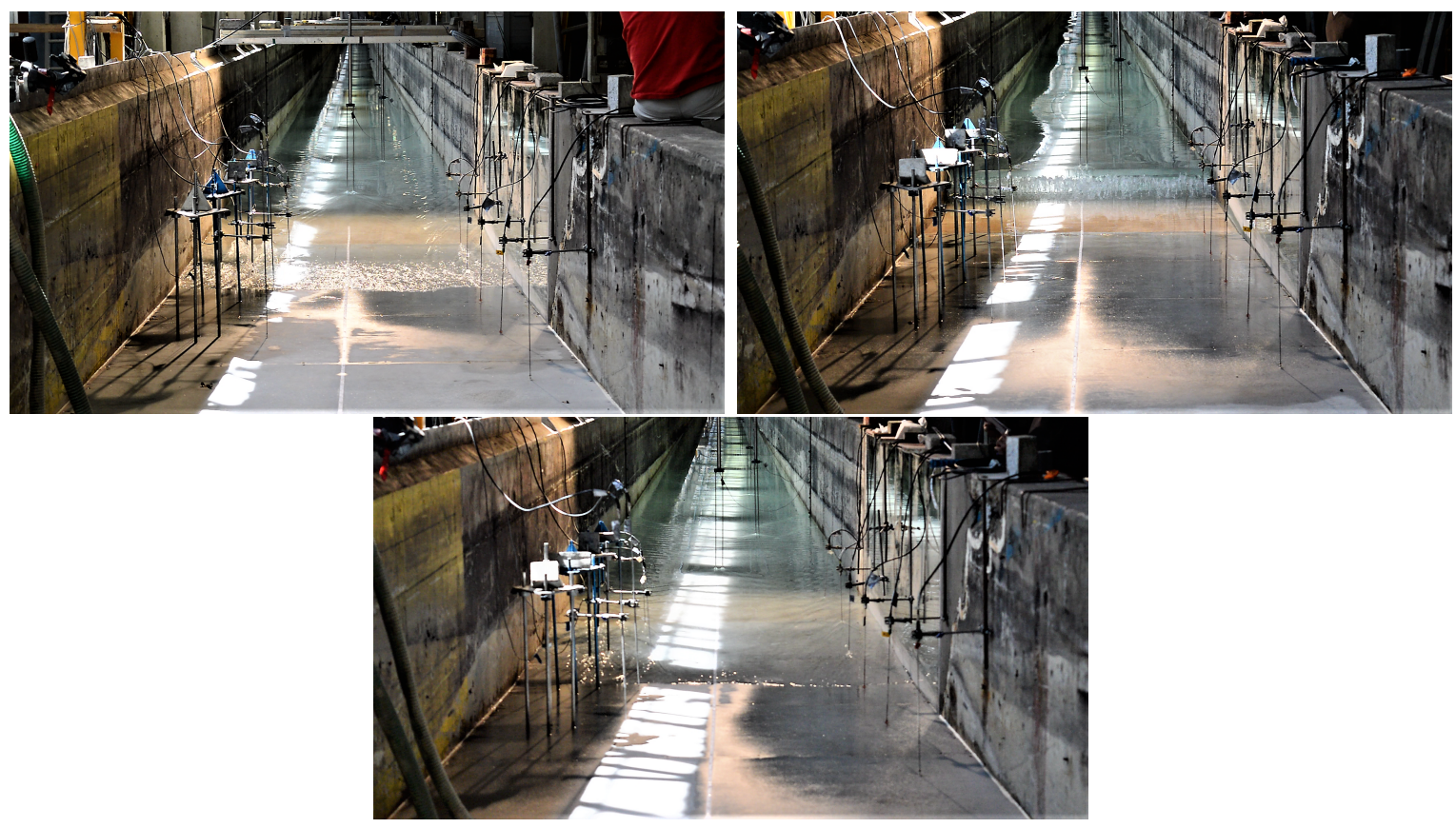

Figure 2: Video image still of: top left) broken surge/collapsing/spilling breaker, top right) plunging breaker and bottom) unbroken surge.

measured along the glass wall side showed no consistent difference in the two measurements. Therefore, the centreline reading is considered sufficient. Typical examples of the wave runup front during the runup process are shown in Figure 2. Velocity profiles are collected at the bathymetry toe using a Nortek Aquadopp 2 MHz High-Resolution Acoustic Doppler Current Profiler (ADCP) which is accurate to within $\pm 0.5 \mathrm{~cm} / \mathrm{s}$. The velocity data is de-spiked using the phasespace method of Goring and Nikora (2002) and is given as $V=\sqrt{u^{2}+v^{2}}$. The $u$ and $v$ components are measured along differently angled beams, however, this does not affect the measurements as it will be shown in $\S 3.2$ that the flow in the flume is strongly two-dimensional and stream-wise.

\subsection{Tested Waveforms}

An extensive suite of elevated (herein refered to as crest-led) and trough-led waves with periods $T \approx 10$ to $230 \mathrm{~s}$ are simulated (Table B.1) including 13 waveforms that are repeated four or more times (Table 2). Figure 3 presents the recorded and theoretical $\eta(X, t)$ for each change in $T$ in the repeated wave set. The waves differ from the mathematical description of solitary and $N$-waves. The theoretical trough of an $N$-wave is generally shorter in length and steeper than the recorded trough-led waves while the recorded crest-led waves are not as 
steep as an equivalent height solitary wave. However, studies have shown that real tsunami waveforms do not follow idealised cases and are made up of a number of incident and reflected waves (Grilli et al., 2013).

The calibration for the crest-led waves uses the solitary wave solution for the C25 wave $(T=25 \mathrm{~s}$, Figure $3 \mathrm{k})$ as the target, with which the measured wave fits well. To achieve larger crest-led wave periods, the wave shape is elongated to the desired period while retaining the largest amplitude possible given the finite volume capacity of the PLWG. This leads to smaller amplitudes for increasing wave periods and a wave shape that departs from the theoretical solitary wave profile but is reasonably closer to a real tsunami profile (for example, Figure A.1b-d). For trough-led waves, the calibration fits the short period 40s wave to the mathematical solution for an equivalent period $N$-wave as closely as possible (Figure 3a). While the fit is reasonable, the measured time-series more closely follows a sine function (where $\eta(t)=a^{+} \sin (2 \pi f t)$, Figure 3f). For longer trough-led waves, the waves are elongated to produce the desired period with the maximum possible amplitude. The resulting fits with Equation (2) are not as good, while the sine function shows a reasonable fit. The exception is for TL80d, which represents the largest amplitude possible for a period which has relevance to tsunami. This results in a reduction of the available volume in the PLWG to generate a trough of symmetrical negative amplitude to the crest, as more volume is initially taken up to produce the large crest.

The wave characteristics are defined at $X=65.6 \mathrm{~m}$. For trough-led waves, $T$ is calculated from the difference between time at the start of the trough $t_{\text {start }}$ and the end of the crest $t_{\text {end }}$ (Figure 4a). $t_{\text {start }}$ and $t_{\text {end }}$ are respectively defined as the times of the first and second down-crossings of $\eta(X, t)$ across the value corresponding to $1 \%$ of the maximum positive amplitude $a^{+}$. The maximum negative $\eta(X, t)$ defines the negative amplitude $a^{-}$. For elevated waves $t_{\text {start }}$ and $t_{\text {end }}$ are defined as the times when $\eta(X, t)$ first up-crosses and then first down-crosses the value corresponding to $1 \%$ of $a^{+}$respectively (Figure $4 \mathrm{~b}$ ). Celerity $C_{\text {exp }}$, is calculated from the temporal correlation of the beginning of the waveform between the last offshore wave gauge $(X=47.0 \mathrm{~m})$ and the bathymetry toe wave gauge $(X=65.6$ $\mathrm{m})$. The wavelength is defined as the product of celerity and period $\left(\lambda=C_{\exp } T\right)$. There are discrepancies between the recorded $C_{\exp }$ and theoretical $C=\sqrt{g d}$ indicating non-linearity in the generated waves (Table 2, the full range of wave conditions and variables are given in Table B.1). Referring to the solution regions described in Hedges (1995), the waves tested lie within the cnoidal theory demarcation, suggesting linear wave theory may not be fully 
applicable to these waves.

Table 2: Characteristics of the wave conditions that are repeated four or more times defined at $X=65.6 \mathrm{~m}$ where 'TL' and ' $\mathrm{C}$ ' denote trough and crest led waves respectively. The full range of wave conditions tested is provided in Table B.1.

\begin{tabular}{|c|c|c|c|c|c|c|c|c|}
\hline $\begin{array}{l}\text { trough/ } \\
\text { crest-led } \\
\text { TL/C }\end{array}$ & $\begin{array}{l}T \\
(\mathrm{~s})\end{array}$ & $\begin{array}{l}\lambda \\
(\mathrm{m})\end{array}$ & $\begin{array}{l}a^{+} \\
(\mathrm{m})\end{array}$ & $\begin{array}{l}a^{-} \\
(\mathrm{m})\end{array}$ & $\begin{array}{l}d \\
(\mathrm{~m})\end{array}$ & $\begin{array}{l}H / d \\
(\mathrm{~m})\end{array}$ & $\begin{array}{l}C_{\exp } \\
(\mathrm{m} / \mathrm{s})\end{array}$ & $\begin{array}{l}\text { difference from } \\
\sqrt{g d}(\%)\end{array}$ \\
\hline TL230 & 230 & 560 & 0.038 & -0.041 & 1 & 0.08 & 2.43 & 31 \\
\hline TL180 & 184 & 656 & 0.043 & -0.042 & 1 & 0.09 & 3.58 & -13 \\
\hline TL160 & 161 & 492 & 0.043 & -0.040 & 1 & 0.08 & 3.06 & 1 \\
\hline TL110 & 108 & 403 & 0.055 & -0.044 & 1 & 0.10 & 3.71 & -15 \\
\hline TL80a & 79 & 226 & 0.030 & -0.030 & 1 & 0.06 & 2.85 & 12 \\
\hline TL80b & 82 & 268 & 0.044 & -0.040 & 1 & 0.08 & 3.25 & -5 \\
\hline TL80c & 81 & 283 & 0.060 & -0.001 & 1 & 0.11 & 3.49 & -11 \\
\hline TL80d & 81 & 245 & 0.080 & -0.061 & 1 & 0.14 & 3.04 & 4 \\
\hline TL40 & 39 & 176 & 0.060 & -0.045 & 1 & 0.11 & 4.50 & -30 \\
\hline $\mathrm{C} 25$ & 24 & 69 & 0.083 & $\mathrm{~N} / \mathrm{A}$ & 1 & 0.08 & 3.56 & -13 \\
\hline $\mathrm{C} 45$ & 44 & 113 & 0.064 & $\mathrm{~N} / \mathrm{A}$ & 1 & 0.06 & 3.46 & -11 \\
\hline C80 & 83 & 193 & 0.069 & $\mathrm{~N} / \mathrm{A}$ & 1 & 0.07 & 2.58 & 20 \\
\hline $\mathrm{C} 200$ & 202 & 558 & 0.057 & $\mathrm{~N} / \mathrm{A}$ & 1 & 0.06 & 2.76 & 12 \\
\hline
\end{tabular}



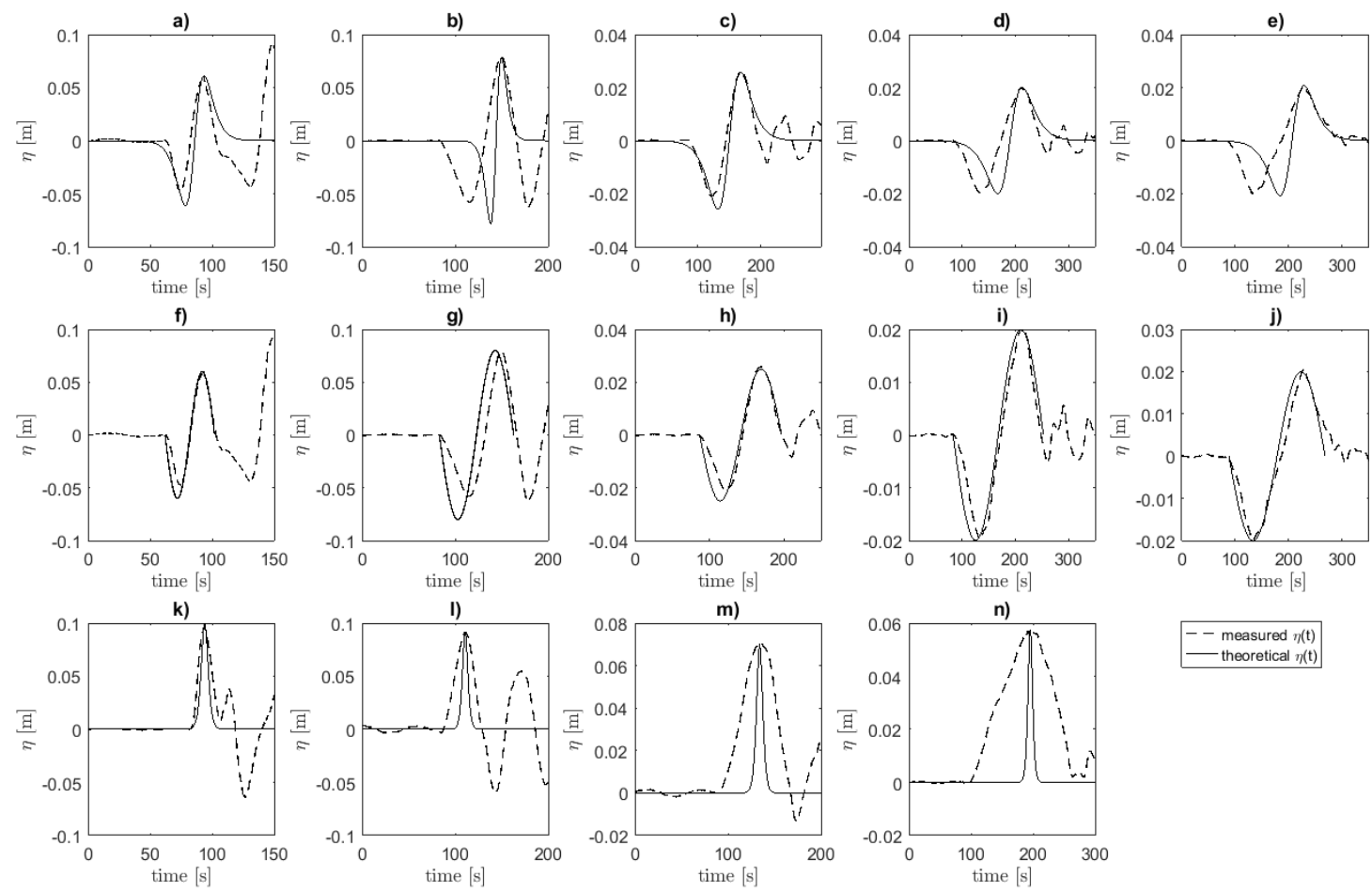

Figure 3: The recorded time-series $\eta$ for runs of the TL40, TL80d, TL110, TL160, TL230, C25, C45, C80 and C200 waves, along with the mathematically described $\eta(t)$ signal. The trough-led waves are compared with the $N$-wave Equation (2) (Figure 3a-e), the sine function $\eta(t)=a^{+} \sin (2 \pi f t$ ) (Figure 3f-j) and the crest-led waves with the solitary wave Equation (1) (Figure 3k-n). 


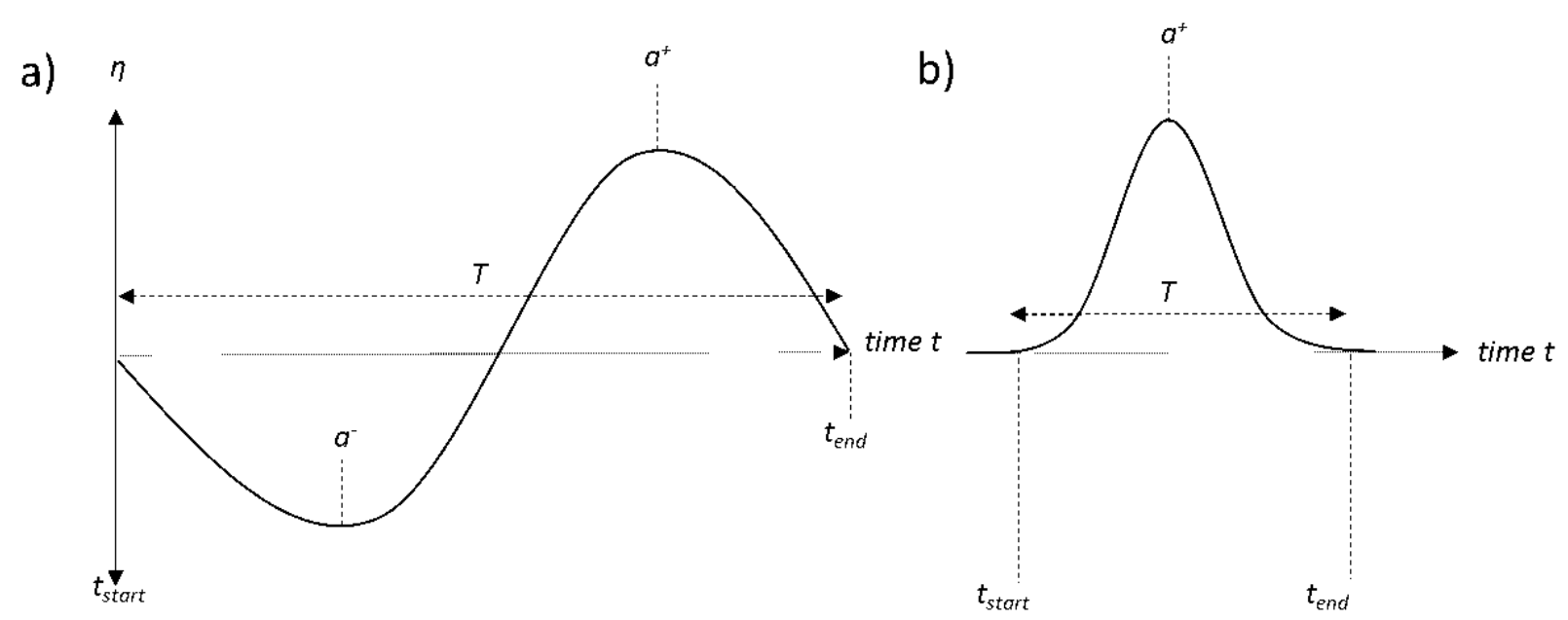

Figure 4: Schematic of the definitions of a) trough-led and b), crest-led wave characteristics including period $T$, positive and negative amplitudes $a^{+}$and $a^{-}$respectively, and $t_{\text {start }}$ and $t_{\text {end }}$.

\subsection{Repeatability}

To ensure repeatability of the waveforms and the inferences made from the observations, the waves listed in Table 2 are repeated at least four times. The mean and standard deviation $(\sigma)$ of the $a^{+}, a^{-}, C_{\text {exp }}, \lambda$, runup $(R)$ and the potential energy ( $E_{p}$, defined using Equation (4)) of each repeated waveform is also reported. Standard deviation is small for all parameters, and of the same order of magnitude as the error in the runup measurement, with the exception of $T$ where the variation is slightly higher. This indicates that the experimental set-up and data is repeatable.

$$
E_{p}=\int_{0}^{t} \frac{1}{2} \rho g \eta(t)^{2} C_{\text {exp }} \mathrm{d} t
$$

\subsection{Wave Bore Formation}

The qualitative presence of wave breaking and consequent bore formation is determined from analysis of video images of each wave (Figure 2a-c). Breaking of waves of $T \approx<20$ s is easy to record due to the observation of white water. The presence of breaking for waves of $T \approx 20$ - 40s is less easy to define and no comment can be made on the transition of periods between a collapsing breaker and an unbroken surge. The primary focus of this paper is on periods much greater than $40 \mathrm{~s}$ (Table 2), where breaking does not occur (as observed visually) in the waves tested. Discussions of breaking in this paper are purely qualitative; future research will need to corroborate with quantitative and repeatable analysis of breaking. 


\subsection{Scale Considerations}

For engineers to have confidence in their use, it is important that physical test facilities produce prototype-scalable wave characteristics. When modelling free-surface phenomena such as waves, Froude scaling is often preferred as gravity is the main restoring force both in the model and prototype (Hughes, 1995). Froude similitude in scaling requires the Froude number $\mathrm{Fr}=U / \sqrt{g d}$ (where $U$ is a characteristic velocity), is the same in the model and prototype. However, it is important to address the effect of the chosen similitude in the Froude number over the Reynolds and Weber numbers. The Reynolds number $R e=C d / \nu$ (where $\nu$ is the kinematic viscosity), describes the importance of viscous effects. The Weber number, $W e=\rho \nu^{2} l / \sigma_{s}$ (where $\rho$ is density of water, $l$ is a characteristic length, and $\sigma_{s}$ is the surface tension), describes the importance of surface tension effects. In these tests (Table B.1) the minimum and maximum values of $R e$ are $1.7 \times 10^{6}$ and $4.5 \times 10^{6}$ respectively, both which describe fully turbulent conditions (Hughes, 1995). The minimum and maximum We numbers for these experiments (using $C$ and $d$ as the characteristic velocity and length, Table B.1) are $2.76 \times 10^{4}$ and $5.62 \times 10^{4}$ respectively. This indicates that in the constant depth region of the flume the scale effects from $R e$ and $W e$ are negligible, and that $F r$ similitude is appropriate.

Drähne et al. (2016) discuss scale effects on long wave runup in detail, and much of their analysis applies to the current test set up. During the runup process, Re is defined by the local water depth and flow velocity, both of which eventually approach zero as runup approaches its maxima. Re becomes small in the nearshore regions and at the leading front of the wave, particularly near the maximum runup (this is particularly apparent in the unbroken leading wave front in Figure 2 bottom). This may increase the influence of viscous effects in the model against the prototype. Drähne et al. (2016) suggest a critical threshold of $R e_{\text {crit }}=10^{3}$ is likely suitable for long wave runup experiments. Thus, here as in their experiments, $R e$ is sometimes less than $R e_{\text {crit }}$ meaning viscous forces may be larger in the model than the prototype.

Weber number dissimilitude also has potential to add error in that the surface tension may become overly influential in the model. Peakall and Warburton (1996), who review the influence of $W e$ in small scale models recommend a threshold between 2.5 to 160 . In this range the flow depth becomes so small that surface tension becomes important. This may occur at the wave front as discussed above for viscous effects. The counteraction of surface tension effects against the inertial forces that drive runup at the wave front may cause an 
underestimation of runup in the model.

The conclusion is, as also discussed by Drähne et al. (2016), that while the model may contain bias from increased viscous and surface tension effects, these are likely negligible against other inaccuracies and assumptions such as slope topography and wave idealisations. Heller (2011) reviews scale effects in physical modelling and shows that for long wave modelling an accepted maximum model scale to measure the dynamic and kinematic parameters is around 1:50. For tsunami $T(\approx 90$ to $7000 \mathrm{~s})$, a 1:50 scaled wavelength is in the order of hundreds of metres. Therefore, to generate such wavelengths either very long flumes are required or an understanding of wave re-reflection is required (as discussed in $\S 3.1$ ).

\section{Analysis of the Generated Waveforms}

The first aim of this paper is to demonstrate the successful generation of scaled tsunamilength waves in the flume. This section focuses on four trough-led waveforms; the TL80d, TL110, TL160 and T180 (Table 2), in order to highlight features of the wave generation and propagation in the flume. The following analysis is carried out; 1), demonstration of the evolution of the waveform with time and discussion of the re-reflections, 2) analysis of the waveform as it propagates up the bathymetry and 3) analysis of the waveform velocity profiles.

\subsection{PLWG Waveform Propagation and Reflection}

As the wavelengths of the four waveforms are in the range of 2.7 to 6.2 times the length of the flume (90m, Table 2), it is not possible to generate and propagate the entire waveform in the flume. To visualise the wave propagation in the flume Figure 5 shows the variation in $\eta$ as a function of time (x-axis) and distance along the flume length (y-axis). The figure shows a clear decrease in $\eta$ at the PLWG $(X=0)$ and the propagation of this draw-down towards the beach. Once the draw-down is complete the wave crest can be seen at the PLWG and propagating down the flume with time. These results show that the wave running up the bathymetry is made up primarily of the incident wave. For the purpose of this study only the initial part of the wave is of interest, after which evidence of a standing wave pattern can be observed, particularly in Figure 5a-b.

Wave reflection interference can be clearly observed in the central portions (Figure 5a, $t \approx 70 \mathrm{~s}$ and $X \approx 30 \mathrm{~m}$ ) and beginning of the flume (Figure $5 \mathrm{~b}, t \approx 50 \mathrm{~s}$ and $X=0 \mathrm{~m}$ ) and after the main event has occurred. They manifest as destructive and/or constructive 
interference on the incoming wave. Interference is the net effect on the free surface of the superposition of the reflected and incident wave. The reflection occurs as the incoming wave reflects off the sloping bathymetry (see e.g., Hughes 1995). This natural reflection propagates back until (conservatively), it hits the leading edge of the PLWG flow shaper and will re-reflect back into the flume. The re-reflections are a source of error and require minimisation. In the present situation destructive interference causing a net decrease in $\eta(X, t)$ occurs when the re-reflected trough interacts with the incoming wave crest. The opposite is true of constructive interference, where the re-reflected wave is above the still water level resulting in a net increase in $\eta(X, t)$. By calculating the time of the re-reflection from $C_{\text {exp }}$, the appearance of interference on $\eta(X, t)$ can be determined (McGovern et al., 2016). In this flume at the bathymetry toe the interference caused by the natural reflection is generally constructive only.

Figure 6 shows the waveforms $\eta$ normalised by the positive amplitude $a^{+}$of the wave at $X=65.6 \mathrm{~m}\left(a^{+}\left(X_{t o e}\right)\right)$ at the different positions on the sloping bathymetry $(X \approx 65$ to 84 $\mathrm{m}$ ) as a function of $t / T$, (where $t=$ instantaneous time from the start of the waveform). The waveforms have been shifted to enhance the visualisation and comparison of the free surface profiles. The results show that the waveform is generally preserved over the propagation distance $(\approx 20 \mathrm{~m})$. There are more pronounced changes for the longer waveforms (Figure $6 \mathrm{~d})$ where the position of the superimposed short period waves evolves. This has an impact on determining the correct amplitude of the wave. However, these small oscillations are a magnitude smaller than the incident wave and despite this shortcoming the results appear reasonable. In addition the results show that the amplitudes of the crest $\left(\left|a^{+}\right|\right)$and trough $\left(\left|a^{-}\right|\right)$increase as the wave moves up the bathymetry, (see Figure 7 ). This shoaling effect appears linear apart from Figure $7 \mathrm{~d}$ where the crest amplitude is effected by the secondary superimposed waves. This is less of an issue for the trough. The linear increase in amplitude demonstrates that the destruction from the re-reflection of the wave trough is negligible.

For these very long waves, the interference of reflection on the waveform at earlier positions in the flume has important implications on the definition of wave amplitude used in various runup prediction methods, and will be further discussed later $(\S 4.3)$. The bathymetry toe is chosen as it delineates a definitive change in the bathymetry slope that could be easier to relate to prototype than an arbitrary position offshore over a constant depth of arbitrary length. Due to the long wavelength of the waves being considered, they are composites of both the incident and reflected components at any point in the flume. Therefore, by choosing 

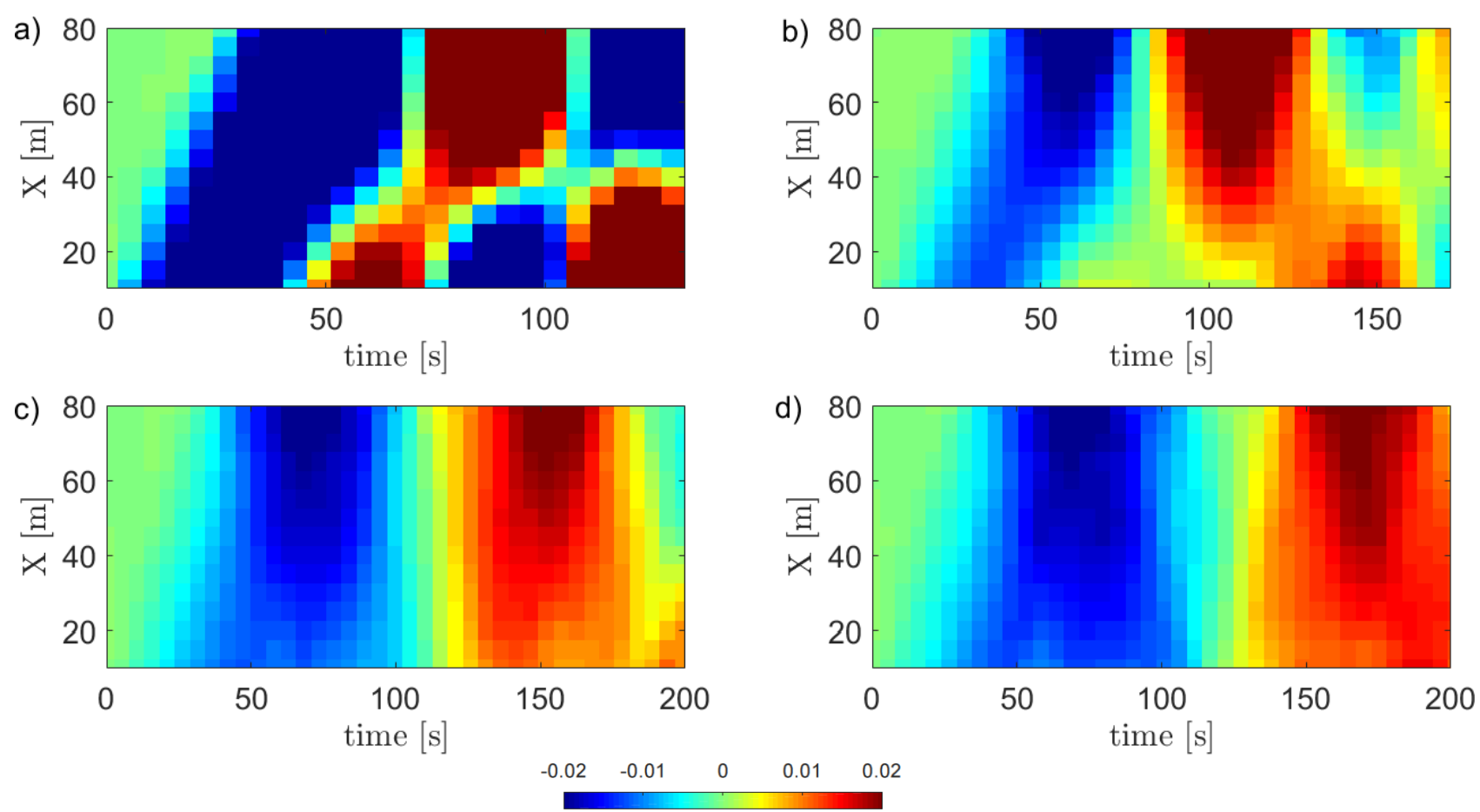

Figure 5: Hodograph plot of the evolution of the waveform in the flume in increments of $5 \mathrm{~s}$ for a) TL80d, b) TL110, c) TL160 and d) TL180. Evolution of $\eta$ as a function of time (x-axis) and distance ( $y$-axis). Colour bar scale in $[\mathrm{m}]$.

to define at the bathymetry toe the waveform may be defined with the immediate effects of reflections from the slope of given length and angle in front of it accounted for. The alternative being a wavelength dependent reflection that may be constructive or destructive to varying degrees depending on both the incident wavelength and the position of definition away from the reflecting slope. Further, the offshore region of the flume acts as a buffer zone for the wave to stabilise and for short frequency non-linear effects of the outlet to dissipate, leaving a smooth waveform. This length is not long enough for the $T \approx 200 \mathrm{~s}$ wave to fully stabilise. However, the small amplitude superimposed wave, whose period $\sim 22 \mathrm{~s}$, may also be in part attributed to an excitation of the 2 nd harmonic of the flume's resonant frequency, estimated at $44-49 \mathrm{~s}$, (Chandler et al., 2016). Its growth in amplitude with increasing $X$ may be due to a combination of energy transfer between the long wave and the short waves and/or shoaling.

The negligible presence of destructive and constructive re-reflections as discussed in this section demonstrates the absorption of the re-reflection by the PLWG. This occurs through adjustments of the control variable (the valve angle as a function of time, $\theta(t)$ ) in an effective 

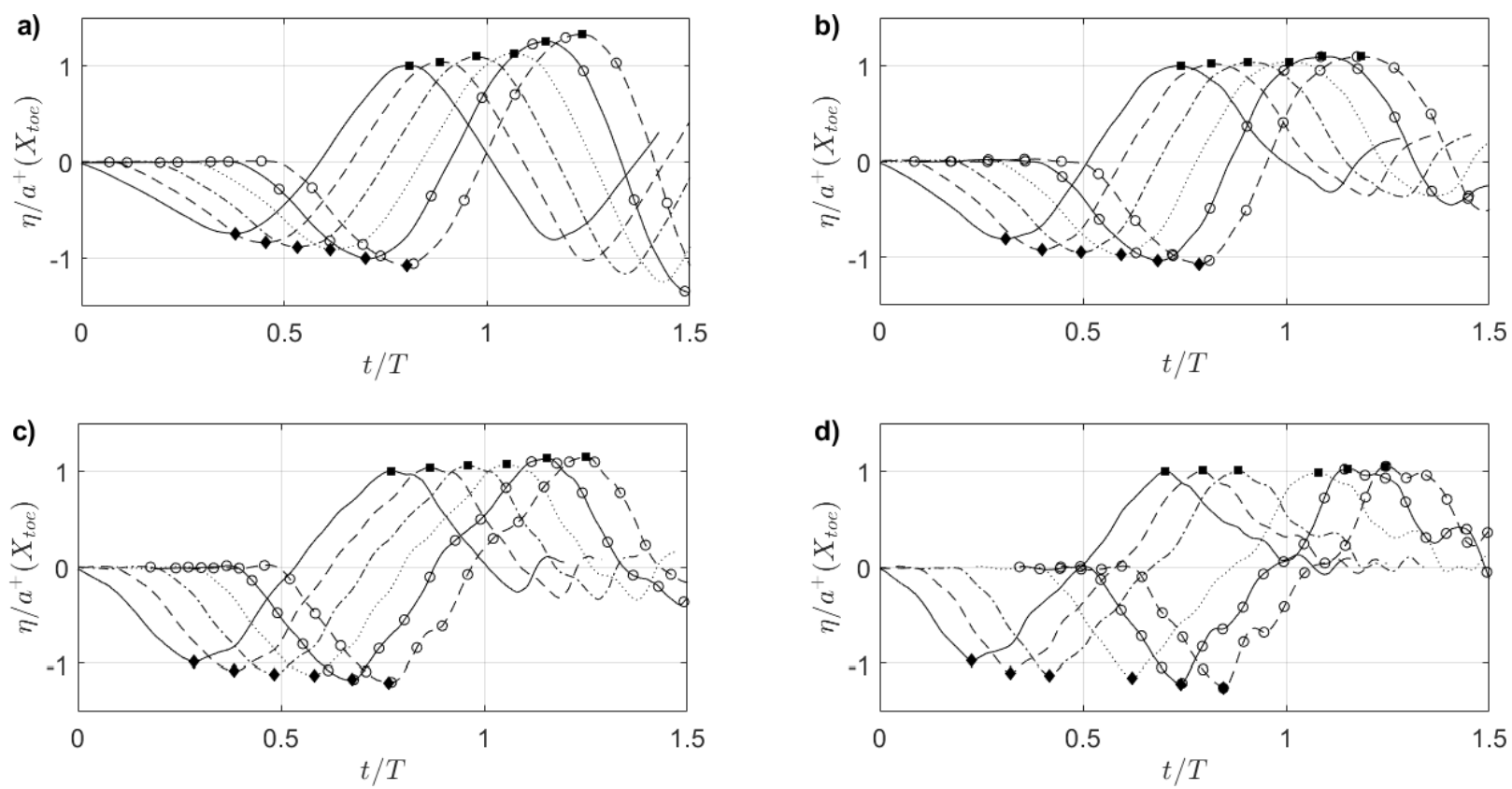

Figure 6: Non-dimensional waveform, $T=$ a) TL80d b) TL110, c) TL160 and d) TL180, on the bathymetry $\left(X=-65.6 \mathrm{~m},-70.6 \mathrm{~m},-.73 .6 \mathrm{~m},: 76.6 \mathrm{~m},-(\circ) 81.6\right.$ and $-(\circ) 83.6 \mathrm{~m}$ ) where $a^{-}$is marked with $\diamond$ and $a^{+}$ as $\square$.

a)

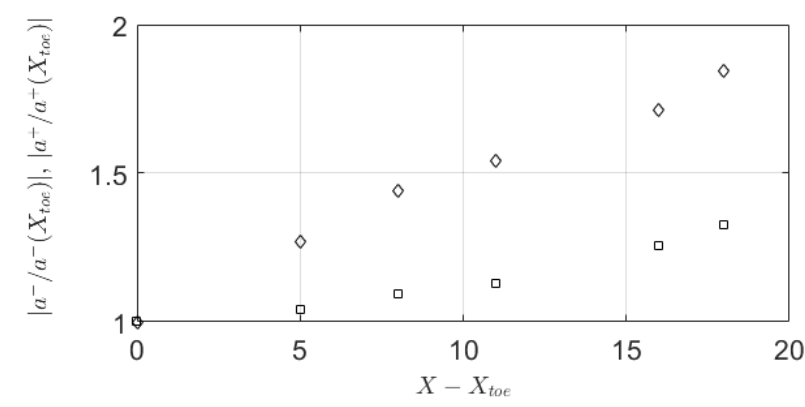

c)

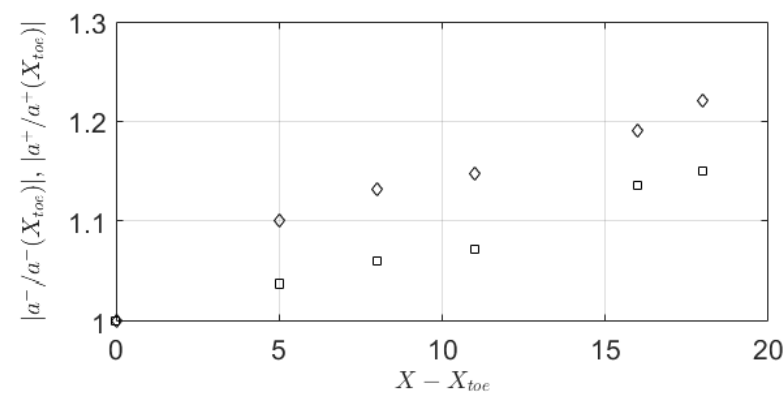

b)

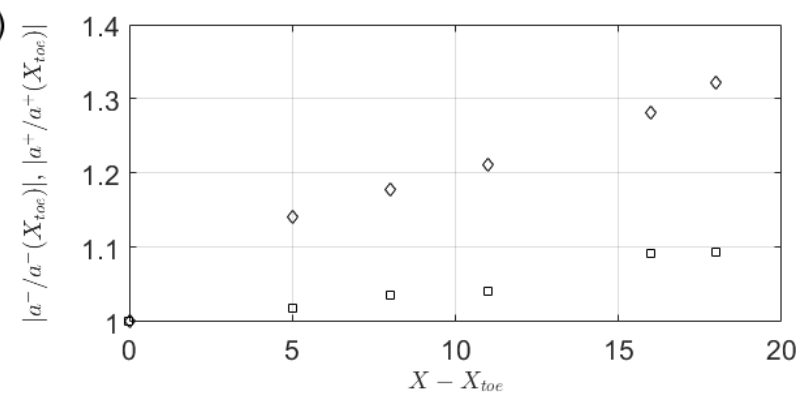

d)

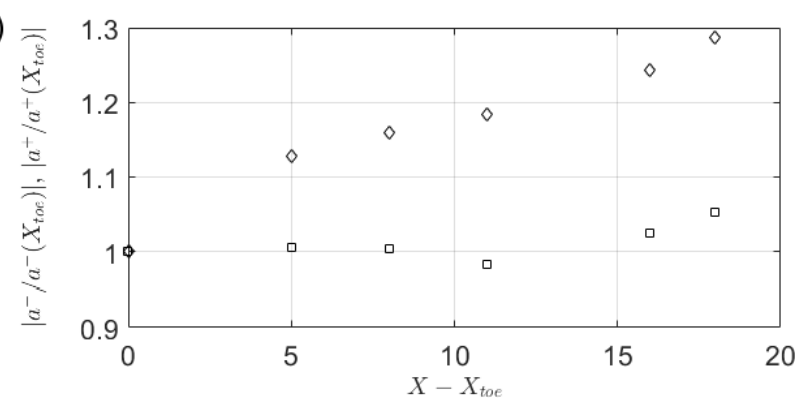

Figure 7: Increase in magnitude of the trough amplitude $\left|a^{-}\right|$and crest amplitude $\left|a^{+}\right|$shown as $\diamond$ and $\square$ respectively for a), the TL80d, b) the TL110, c) the TL160 and d) the TL180. 
open-loop absorption method to attain the desired $\eta(X, t)$ while minimising second order wave reflections. Such adjustments are carried out iteratively over the calibration process for each target waveform. This appears a similar solution to that of Bremm et al. (2015), in which the input signal for the control variable is pre-calibrated. For the PLWG, the calibration process may take several hours depending on the desired waveform.

\subsection{Velocity Profiles}

To ascertain whether the PLWG generates waves with expected water flow characteristics, velocity profiles are measured at the bathymetry toe using the ADCP. Very long shallow water waves should manifest highly elliptical (nominally horizontal) fluid particle motions over the full water depth. The position from the PLWG at which the velocity profiles are recorded $(X=65.6 \mathrm{~m})$ is expected to be beyond that of which evanescent wave modes that are attached to the PLWG are present. This is demonstrated in Figure 8 where the regular $8 \mathrm{a}-\mathrm{d}$ ) and logarithmic velocity profiles (which are zoomed in on the lowest $0.2 \mathrm{~m}$ of the water column 8e-h) for each wave are given. The gap between $Z=0$ and the first data point is due to the down-looking instruments blanking distance. The instrument cell size, number and range is changed to suit each wave condition separately, leading to different profile sizes and lengths on Figure 8. Additionally in Figure 8i-1 the $\eta(X, t)$ at $X=65.6 \mathrm{~m}$ is given as a function of $t / T$. The negative values of $V$ denote flow direction towards the PLWG.

The profiles for all waves are generally constant with $Z$ except near the bed where boundary layer effects are observed. The boundary layer profiles do not always fit the the log-law profile, particularly at low velocities. Those that do are generally for larger velocities. The direction of flow corresponds to the propagation of the wave. Starting at $t / T \approx 0$ flow becomes negative (towards the PLWG) until the base of the trough. As flow returns from the PLWG it becomes positive until after the crest when negative flow returns and flow recedes back towards the PLWG. Peak velocities are out of phase with the trough and crests (occuring before them) suggesting that linear wave theory does not describe the generated wave particle motions well. As seen above in regards to wave celerity (Section $\S 2.2$ ), the solution regions described in Hedges (1995) suggest that these waves may lie within the Cnoidal theory. (ASCE/SEI, 2017) suggest the overland peak flow velocity to occur before the maximum flow depth, matching this observation from the offshore region. Further examination of this will be attempted in future work. 

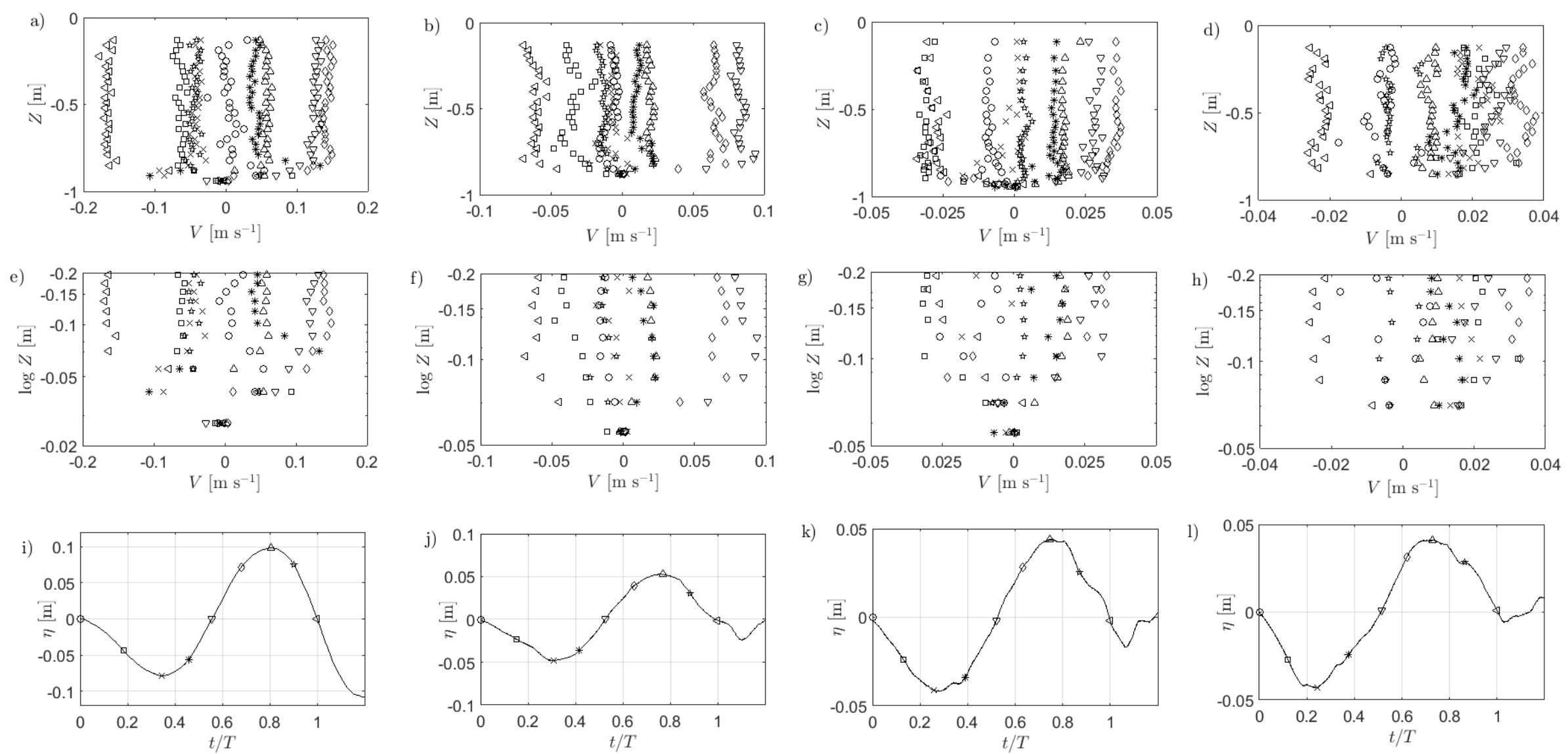

Figure 8: a-h Regular and logarithmic (lowest $0.2 \mathrm{~m}$ of water column only) velocity profiles showing $V(Z, t)$ for TL80d (a,e), TL110 (b,f), (c,g)

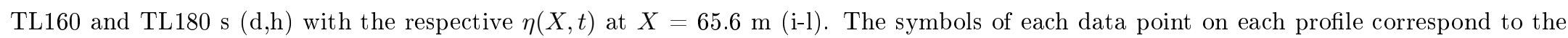
symbols on the respective $\eta(t)$ plot indicating the value of $\eta(X, t)$ the velocity profile corresponds to. 
A weakness of the first generation PLWG was that the abruptness of the tank outlet flow generated significant energy losses and eddying. Schimmels et al. (2016) argue that due to these weaknesses 1) there remains uncertainty in the total hydrodynamics of the whole waveform in the flume at any given time and 2), lack of well-defined boundary condition renders the validation of numerical models with PLWG data difficult. Non-linearities at the tank outlet are corrected in the design of the 2nd generation PLWG through the use of a flow shaping device (Figure 1 and Allsop et al. 2014). Its effectiveness is shown in Figure 9, which shows the maximum negative and positive $V(Z, t)$ for six runs of the TL80d wave as recorded near the PLWG outlet at $X=5.85 \mathrm{~m}$ and $Y=0,0.3$, and $0.6 \mathrm{~m}$. The two-dimensionality and repeatability of the profiles demonstrate a smooth flow at the outlet is present and for these waves the flow at both the outlet and at the toe is well-defined.

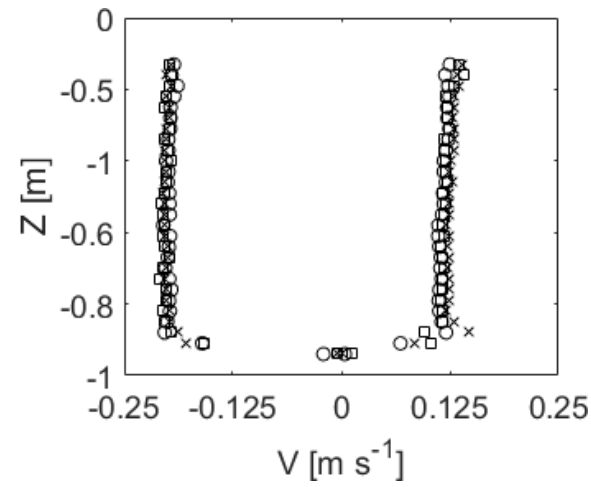

Figure 9: Velocity profiles showing the approximate maximum $V(Z, t)$ for repeats of TL80d recorded at $X$ $=5.85 \mathrm{~m}$ and $Y=0(\square), 0.3(\times)$ and $0.6(\circ) \mathrm{m}$. Positive and negative values denote flow direction away and towards PLWG respectively.

In summary, $\S 3$ analyses the generation and propagation of tsunami-length waves by the PLWG. The discussion of the waveform propagation and reflection in $\S 3.1$ shows that the presence of re-reflections are negligible in the near-shore region. The analysis of the velocity profiles in $\S 3.2$ confirms the inherent two-dimensionality of the laboratory set-up and that flow of water corresponds to the waveform generated. Three reasons are discussed for selecting the definition point for the wave at the bathymetry toe. First, the bathymetry toe delineates a definitive change in slope and is more readily definable geographically at full scale than an arbitrary position offshore over a constant depth of arbitrary length. Second, it allows the wave to be considered with the consistent reflection caused by the adjacent slope, rather than a wavelength dependent reflection that may be constructive or destructive 
to varying degrees depending on both the incident wavelength and the position of definition away from the reflecting slope. Finally the definition of the wave at the bathymetry toe also reduces possible PLWG outlet flow non-linearities caused by turbulence generated at the PLWG-flume interface manifesting on the defined wave by allowing the wave to propagate and settle first.

\section{The Runup Behaviour of Waveforms with Periods between 5 - $230 \mathrm{~s}$}

Figure 10 shows the recorded $R / a^{+}$(where $a^{+}=a^{+}\left(X_{t o e}\right)$, the positive amplitude defined at the bathymetry toe) for all the waves tested (as given in Table B.1) as a function of $T$. From $T \approx 100$ and greater, $R / a^{+}$tends to unity. At shorter periods, $R / a^{+}$increases to a maximum of $\approx 5$. The results are now compared with available predictor equations.

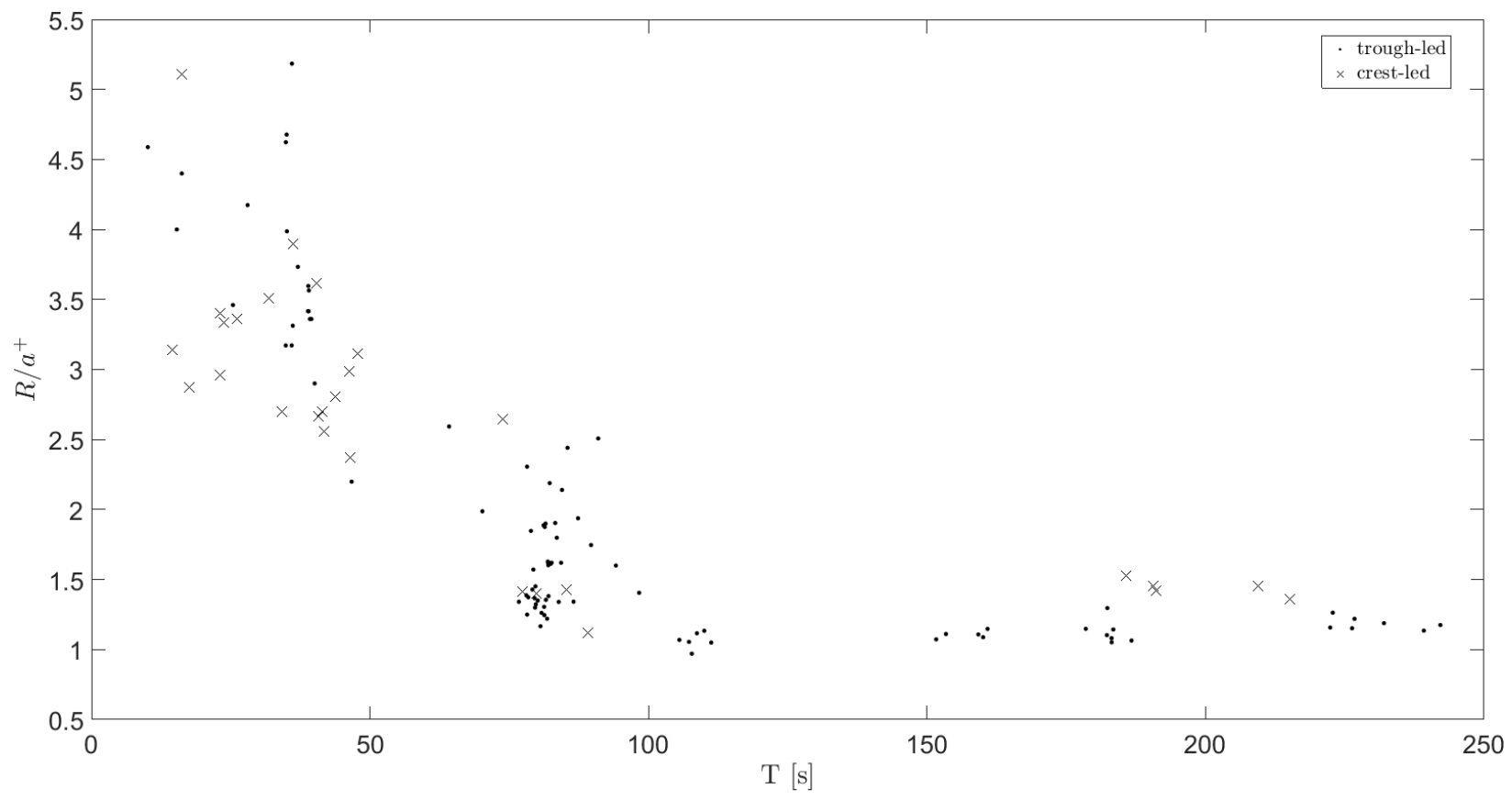

Figure 10: $R / a^{+}$as a function of $T$ for all waves tested.

\subsection{Comparison with available runup predictor equations}

A comparison with available equations in the literature is now made. In some cases this leads to the reported waves, which are composites of the incident and reflected wave, being compared with prediction equations that are based on inccident waves only. These cases will be defined. Figure $11 \mathrm{a}-\mathrm{b}$ presents $R$ normalised with the predicted runup $\left(R_{p}\right)$ versus $T$ for the trough and crest-led waves calculated using the 'long $N$-wave' (Equation 5) and 'long 
elevated wave' (Equation 6) equations proposed by Charvet et al. (2013). In the current experiments $d$ varies from a minimum of 0.46 to a maximum of $1.024 \mathrm{~m}$, and $l_{\text {beach }}$ varies from $15.11 \mathrm{~m}$ to $3.83 \mathrm{~m}$ respectively. From Equation (3) this gives $T_{b}=14.22 \mathrm{~s}$ and $2.4 \mathrm{~s}$ respectively. $T / T_{b}$ is $>1$ for all waves suggesting using $T / T_{b}$ may not be an appropriate delineation. Though it is not stated in Charvet et al. (2013), Equations 5 and 6 are likely based on incident-only wave forms, (approximately $T<10 \mathrm{~s}$ at scale) wave data.

$$
\frac{R}{d}=5.75\left(\frac{E_{p}^{+}}{\rho g a^{+} \lambda d^{2}}\right)^{0.4}
$$

$$
\frac{R}{d}=10.18\left(\frac{\rho g\left(a^{+}\right)^{3}}{E_{p}}\right)^{0.89}
$$

where $E_{p}^{+}$is the potential energy of the wave crest (Equation (4)), in which $\eta$ is replaced by $\eta^{+}$, the positive elevation above SWL corresponding to the wave crest. The non-breaking solitary wave equation proposed by Synolakis (1987) is also compared (Equation (7)). Note that the elevated waves generated by the PLWG are not mathematically defined as solitary waves, (see $\S 1.1)$ and are composites of the incident and reflected waves.

$$
\frac{R}{d}=2.831(\cot \beta)^{\frac{1}{2}}\left(\frac{a^{+}}{d}\right)^{\frac{5}{4}}
$$

Hughes (2004a) develops a method for estimating wave runup using a dimensionless wave parameter representing the maximum depth-integrated momentum flux $M_{f}$. In the case of non-breaking solitary waves, Hughes (2004a) finds an empirical fit to the runup data of Synolakis (1987) for $\cot \beta=2.08$ and Hall and Watts (1953) for $\cot \beta=1.0,2.14$ and 3.73 given by Equation (8).

$$
\frac{R}{d}=1.82(\cot \beta)^{\frac{1}{5}}\left(\frac{M_{f}}{\rho g d^{2}}\right)_{\max }
$$

where the subscript 'max' denotes the maximum value. An empirical equation for estimating the momentum flux of a solitary wave is given in Hughes (2004b) as 


$$
\begin{aligned}
\left(\frac{M_{f}}{\rho g d^{2}}\right)_{\max }= & \frac{1}{2}\left[\left(\frac{H}{d}\right)^{2}+2\left(\frac{H}{d}\right)\right]+\frac{N^{2}}{2 M}\left(\frac{H}{d}+1\right) \\
& \left\{\tan \left[\frac{M}{2}\left(\frac{H}{d}+1\right)\right]+\frac{1}{3} \tan ^{3}\left[\frac{M}{2}\left(\frac{H}{d}+1\right)\right]\right\}
\end{aligned}
$$

Where $M$ is given as

$$
M=0.98\left\{\tanh \left[2.24\left(\frac{H}{d}\right)\right]\right\}^{0.44}
$$

and $N$ as

$$
N=0.69 \tanh \left[2.38\left(\frac{H}{d}\right)\right]
$$

For the trough-led wave data, the 'long $N$-wave' Equation (5) gives the best performance with a favourably conservative overestimation of $R$ for most trough-led waves of $T \sim<65$ $\mathrm{s}$ (Figure 11a). The equation performs poorly for $T \sim>65 \mathrm{~s}$, over predicting $R$ by a factor of $\sim 2-5$. The poor fit might be expected considering the limited period of the trough-led wave data set the equation is based on ( $T=6.5-8.8 \mathrm{~s}$ at model scale), and though it performs reasonably for $T \sim \leq 65 \mathrm{~s}$, these periods are significantly shorter than a prototype tsunami. For the crest-led data, Figure 11b demonstrates Equation (6) under-predicts $R$. The 'very long $N$-wave', 'general $N$-wave' and 'general elevated wave' equations of Charvet et al. (2013) perform very poorly giving large over predictions of $R$ and are not plotted here. These equations, and Equation (6) are based on wave data of shorter periods than the current data. Charvet et al. (2013) tested 11 waves with $T / T_{b}>1$, and the maximum $T$ (at model scale) were $171 \mathrm{~s}$ and $92 \mathrm{~s}$ for the trough and crest-led waves respectively. The limited data set of $T / T_{b}>1$ suggests the validity of the equations for so-called 'very long' elevated and $N$-waves as defined by the parameter $T / T_{b}$ is unclear. Equation (7) over predicts by a factor of up to 3 for $T \sim \leq 50 \mathrm{~s}$ beyond which the over-prediction increases to $\geq 4$. Equation (8) performs generally well for $T \sim \leq 50 \mathrm{~s}$, giving values of $R_{p} / R 0.63 \approx 2$. At greater periods it over predicts $\approx 2.5$ for most waves.

The sharp change in performance of the equations compared in Figure 11a-b occurs around a shorter period $\left(T \approx 65 \mathrm{~s}\right.$ ) than the approximate range of period in which $R / a^{+}$ converges to 1 ( $T \approx 100$, Figure 10 ). This suggests that $T$ may not be the only causal factor in the runup behaviour of the waves. 

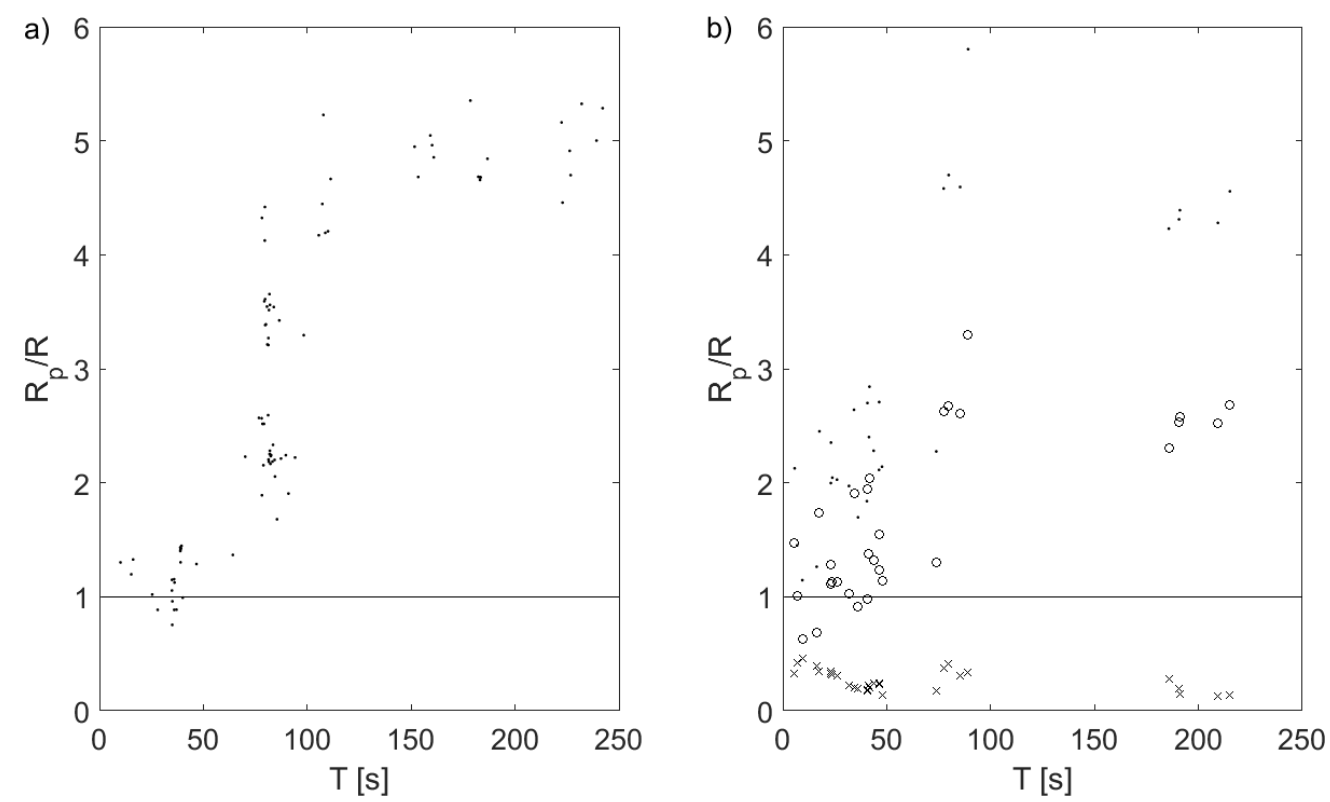

Figure 11: a) Plot of the predicted runup $R_{p}$ using Charvet et al. (2013) Equation (5) normalised with recorded trough-led wave $R$ versus $T$ ('.' symbols). b) $R_{p}$ predicted using Charvet et al. (2013) Equation (6) (' $\times$ ' symbols), Synolakis (1987) non-breaking solitary wave runup Equation (7) ('.' symbols) and Hughes (2004a) non-breaking solitary wave runup equation based on momentum flux Equation (8) ('o' symbols) normalised with recorded crest-led wave $R$ versus $T$

Coastal engineers often characterise $R$ of periodic and transient waves using the Iribarren number $\xi$, (also known as the surf similarity parameter, where $\xi=\tan (\beta) / \sqrt{2 a^{+} / \lambda}$ ), which is a function of the slope of the bathymetry and the wave steepness. Numerous relationships have been derived, including Battjes (1974), Mase (1989), Losada and Giménez-Curto (1981) and in the case of tsunami Madsen and Schäffer (2010). Equations (12a) and (12b), are proposed in the ASCE/SEI (2017) 'Tsunami Loads and Effects' design standard as a means to calculate $R / a^{+}$in the absence of numerical or field data.

$$
\frac{R}{a^{+}}=1.5 \text { for } \xi_{100} \leq 0.6
$$

$$
\frac{R}{a^{+}}=2.50\left[\log _{10}\left(\xi_{100}\right)\right]+2.05 \text { for } \xi_{100}>0.6 \text { and } \leq 6
$$

where $\xi_{100}=$ the Iribarren number defined at the $100 \mathrm{~m}$ offshore depth contour (Equation $(13))$.

$$
\xi_{100}=\frac{T}{\cot (\Phi)} \sqrt{\frac{g}{2 \pi a^{+}}}
$$


where $\Phi$ is the average slope angle from the $100 \mathrm{~m}$ depth contour to the mean high water level along the topographic transect for the site in question.

Madsen and Schäffer (2010) proposed analytical solutions to the non-linear shallow water equations for the runup and rundown of sinusoidal, single waves and isosceles $N$-waves (a symmetrical leading depression $N$-wave). These solutions importantly do not exhibit a tie in between wave amplitude and the horizontal length scale. The solution for an (incident not composite) $N$-wave in terms of $\xi$ is given by Madsen and Schäffer (2010) as

$$
\frac{R}{a^{+}}=X_{\text {elev }} \pi^{\frac{1}{4}}\left(\frac{a^{+}}{d}\right)^{\frac{1}{4}} \xi^{-\frac{1}{2}}
$$

where $X_{\text {elev }}=$ the maximum $/$ minimum shoreline elevation. Values of $X_{\text {elev }}$ for $N$-waves as a function of $\mu$ (where $\mu$ is the amplitude ratio $a^{+} / a^{-}$) are given in Madsen and Schäffer (2010) (Figure 5 therein) in the range of $\mu=0, X_{\text {elev }}=3$ to $\mu=1, X_{\text {elev }}=4.243$ (a perfectly isosceles $N$-wave). For a sinusoidal wave, $X_{\text {elev }}$ is given as \pm 3.5449 .

Figure 12 presents $R / a^{+}$as a function of $\xi$ for the current data set along with Charvet et al.'s (Charvet et al., 2013) and Synolakis' (Synolakis, 1987) data sets. At $\xi>2, R / a^{+}$of Synolakis' solitary wave data deviates from the current data, rising to $\approx 3.5 . R / a^{+}$for the current data decreases to unity at approximately $\xi>2.6$. The curve predicted by Equations (12a) and (12b) is plotted for comparative purposes; it is noted that the current data is scaled to a prototype water depth of $50 \mathrm{~m}$, as opposed to the $100 \mathrm{~m}$ specified by Equations (12a) and (12b). The curve matches Synolakis' data set well but performs very poorly with Charvet et al.'s data set and the current study.

Additionally, Equation (14) is plotted. Using an $X_{\text {elev }}$ value of 1.2 and 1.5 the curves predicted from Equation (14) are given for a non-linearity $\left(\epsilon=a^{+} / d\right)$ value of 0.056 , corresponding to the mean value for the waves in Table B.1. The fit is reasonable, and changing $X_{\text {elev }}$ to larger and smaller values improves the fit at smaller and larger values of $R / a^{+}$respectively. The larger values predicted by Equation (14) may be partially explained by bottom friction effects in the current data, which are not accounted for in the analyitical solution of Madsen and Schäffer (2010). Additionally, for the very long waves the bathymetry slope is effectively seen by the wave as a vertical wall. As the crest of the incident wave moves over the bathymetry toe the (constructive) reflection will approach $100 \%$ of the incoming wave, thereby approaching a doubling the amplitude. It might, therefore, be expected that the use 
of $R / a^{+}$where $a^{+}=$composite positive amplitude will lead to an overestimation of $R$ by Equation (14) of up to a factor of 2.

Drähne et al. (2016) (sinusoidal waves of $T<100 \mathrm{~s}$ ) and Goseberg (2013) (sinusoidal waves of $T 60 \mathrm{~s}$ ) also fit seemingly composite waves to Equation 14, finding a reasonably good match to their data. However, the fit does not match across the whole range of $\xi$ for the current and Charvet et al.'s data.

Though breaking is defined qualitatively in these tests, the different breaking regimes described by the $\xi$ parameter are demonstrated in the current data. Surging waves $(\xi>2.6)$ result in $R / a^{+} \approx 1$, and plunging breakers $(\xi=0.4-2.6)$ result in the larger $R / a^{+}$.

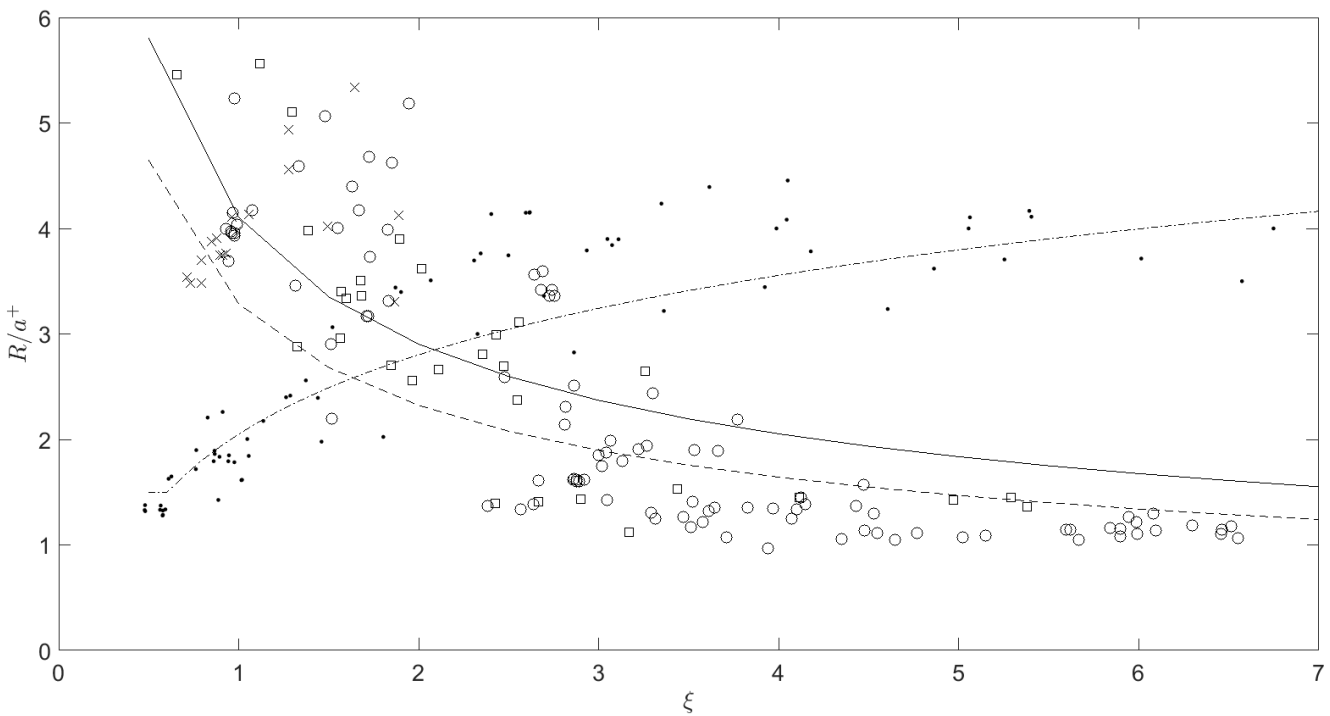

Figure 12: $R / a^{+}$as a function of $\xi$ for the trough-led (o) and crest-led ( $\square$ ) waves tested along with the data from Synolakis (1987) (.), Charvet et al. (2013) $N$-waves $(\times)$, the $\xi$ prediction curves from Madsen and Schäffer (2010) for values of $\epsilon=0.056$ and $X_{\text {elev }}=1.2(--)$ and $X_{\text {elev }}=1.5(-)$ and ASCE/SEI (2017) (-.).

\subsection{Empirical Model for the Runup of Tsunami}

To determine an improved fit to the new long wave data set, the following analysis identifies the explanatory variables that best predict $R / a^{+}$. Correlation plots of $R$ as a function of potentially influencing variables are plotted in Figure 13. A correlation is observed in $T, a^{+}$and $\lambda$. No correlation is observed with $d$, in agreement with Charvet et al. (2013) and interestingly $E_{p}$, contrary to Charvet et al. (2013). Further, while $R$ is not seen to increase when $E_{p}$ increases beyond $\sim 1000 \mathrm{~J} / \mathrm{m}, R$ constantly increases with $a^{+}$. There 

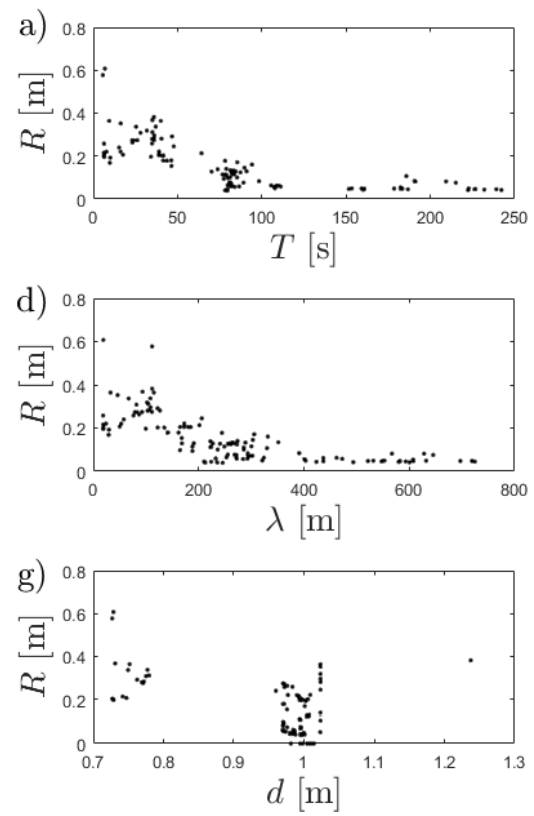
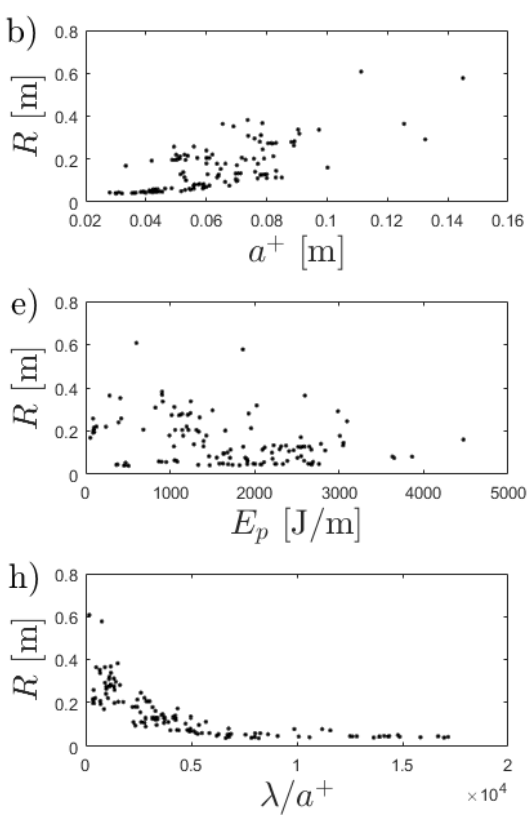
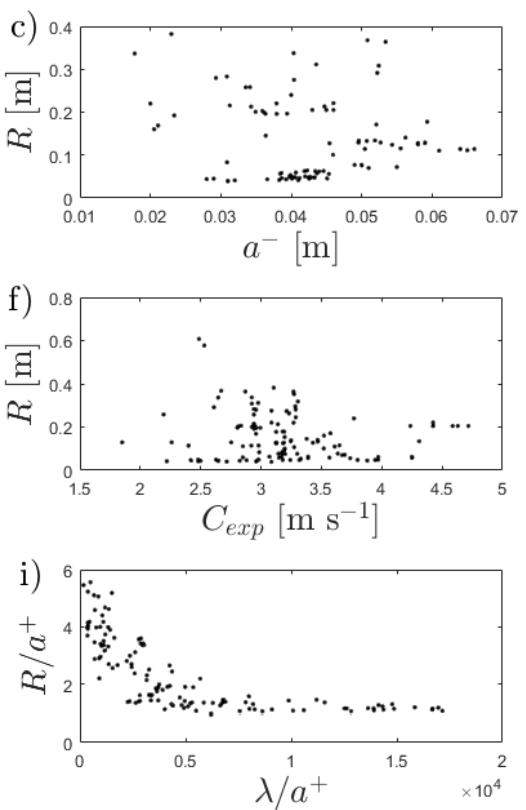

Figure 13: $R$ as a function of a) $T$, b) $a^{+}$, c) $a^{-}$, d) $\lambda$, e) $E_{p}$, f) $C_{\text {exp }}$, g) $d$, h) $\lambda / a^{+}$and i) $R / a^{+}$as a function of $\lambda / a^{+}$for all waves tested.

An empirical fit to the data is now sought for the composite wave data presented. To increase the size of the data set the data of Charvet et al. (2013) is included. The two 
databases consist of 75 unique and independent test conditions. 16 are replicated tests, where PWLG control variable is identical to generate constant $T, a^{+}$and $a^{-}$. These data, whose standard deviation $(\sigma)$ is low (Section $\S 2.3$ ) are not considered independent; the aggregated mean of their measured response variables is considered in the regression analysis.

The response variable $R / a^{+}$may be considered to be a function of $T, \lambda, d, C_{\exp }, E_{p}$ and $\xi$. The 'relative slope length' $\lambda \sin (\beta) / d$ is also postulated as a main controlling variable on $R / a^{+}$and is proposed as a new parameter. As wave steepness has an apparently strong influence on $R / a^{+}$and noting $a^{+}$is the normaliser on Figure $13 \mathrm{i}$ this can be isolated to $\lambda$ alone. $\sin (\beta) / d$ includes information on the wetted length $\left(l_{\text {wet }}\right)$ of the sloping bathymetry the wave travels over by its reciprocal $\left(l_{\text {wet }}=d / \sin (\beta)\right.$, which gives $l_{\text {bathy }} \operatorname{simply}$ as $\left.\sqrt{l_{\text {wet }}^{2}-d^{2}}\right)$. As $\lambda=T C_{\text {exp }}$, and in order to obtain a physically meaningful dimensionless parameter, $\lambda$ is used as the numerator to give the relative slope length parameter $\lambda \sin (\beta) / d$, which describes the ratio of the length of the wave to the wetted length of slope it travels over. Additionally, the product of Iribarren number and relative slope length $\xi(\lambda \sin (\beta) / d)$ is regressed as it includes $a^{+}$, which has a strong correlation to $R / a^{+}$.

There is a sharp transition to unity in $R / a^{+}$for the correlated variables (see correlation plots, Figure C.1a-h). A segmented analysis is used (Hinkley, 1971) as this accounts for sharp changes in the trend of the response variable around an estimated breakpoint. The shape of the statistical model fits the normal distribution of $R / a^{+}$better than the lognormal or gamma distributions, (Figures 14 and C.2). $R / a^{+}$is, therefore, considered to follow a normal distribution related to the explanatory variables in Equations (15a) and (15b).

$$
\frac{R}{a^{+}} \approx(a 1 x+b 1) \text { for } x \geq \text { Breakpoint }
$$

$$
\frac{R}{a^{+}} \approx(a 2 x+b 2) \text { for } x<\text { Breakpoint }
$$

where $a 1, a 2, b 1$ and $b 2$ are coefficients of the fit and $x$ represents the explanatory variable. 


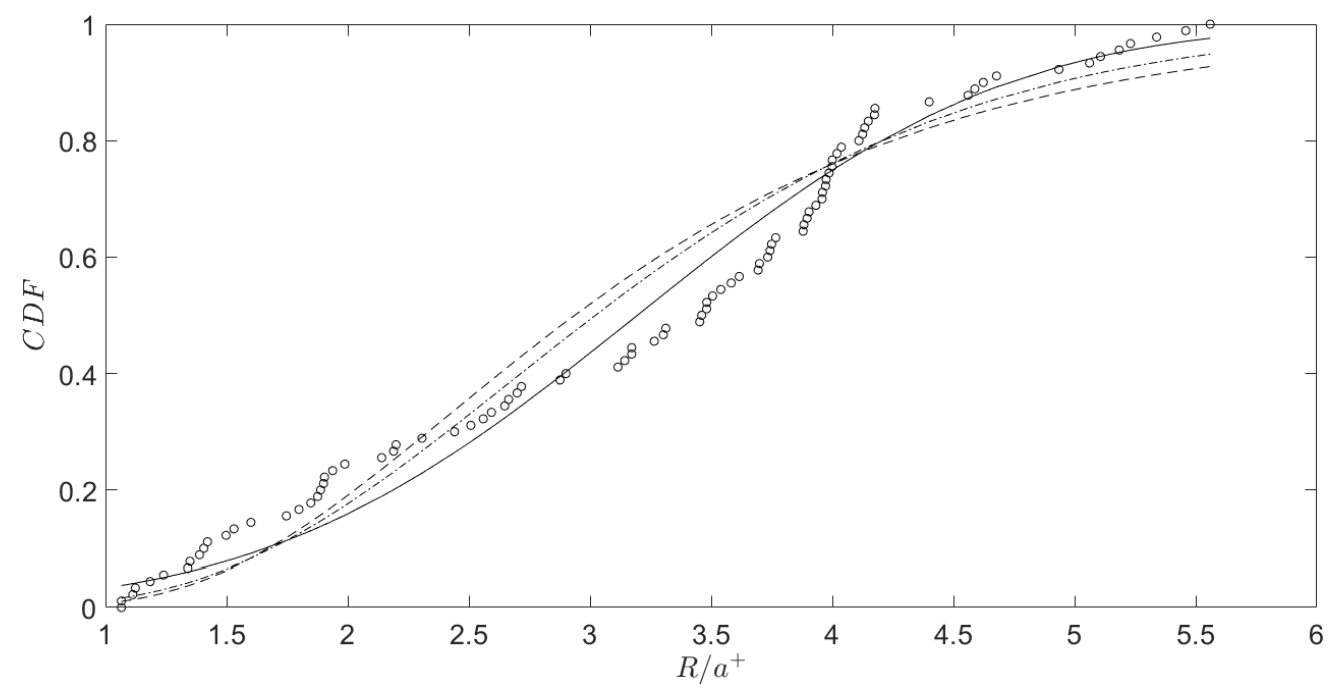

Figure 14: Comparison of the empirical data (o) with the normal (-), lognormal (-) and gamma (-.) cumulative distribution function $(\mathrm{CDF})$ fits to $R / a^{+}$.

It should be noted that $E_{p}$ data is missing for the Charvet et al. (2013) data set, therefore the influencing variables for both the current and the combined data sets are provided separately. The regression models are fitted to the current dataset to assess the importance of $E_{p}$. Both datasets are then used in combination to investigate any change in the results. The Akaike Information Criterion (AIC, Akaike 1974) is used to identify which influencing variable is most capable at describing $R / a^{+}$. A lower value of AIC suggests the variable has a greater influence. The 'segmented' package in the software $R$ (Muggeo, 2008) is used to estimate the parameters of the segmented model, the breakpoint, AIC value and standard error. Table 3 gives the results of the segmented regressions and $R^{2}$ values. The $R^{2}$ is over 0.70 for most cases indicating that most explanatory variables are able to depict a clear trend in the response data. The notable exception is the model which uses $E_{p}$. It can also be noted that the use of data from two databases has a negligible impact on the $\mathrm{R}^{2}$. The question then is which of the used explanatory variables fits the data best, which can be addressed by the use of the AIC. Relative slope length is the most significant explanatory variable for both the current and combined data sets, hence the final formulations of Equations (15a) and (15b) are given by Equations (16a) and (16b) respectively.

$$
\frac{R}{a^{+}}=-0.0364\left(\frac{\lambda \sin (\beta)}{d}\right)+4.553, \quad \text { for } \quad \frac{\lambda \sin (\beta)}{d}<79
$$


Table 3: Results of Segmented Regression

\begin{tabular}{|c|c|c|c|c|c|}
\hline Influencing Variable & Response Variable & Breakpoint & Statistically Significant? & AIC & $\mathrm{R}^{2}$ \\
\hline \multicolumn{6}{|l|}{ Current Data Set } \\
\hline$T$ & \multirow[t]{6}{*}{$R / a^{+}$} & 81.28 & Yes & 112.7 & 0.77 \\
\hline$\lambda$ & & 255.8 & Yes & 113.8 & 0.77 \\
\hline$\xi$ & & 0.655 and 3.521 & Yes & 127.1 & 0.72 \\
\hline$E_{p}$ & & 1,871 & Yes & 156.4 & 0.49 \\
\hline$\lambda \sin (\beta) / d$ & & 68.76 & Yes & 106.5 & 0.79 \\
\hline$\xi \lambda \sin (\beta) / d$ & & 171.8 & Yes & 110.7 & 0.78 \\
\hline \multicolumn{6}{|c|}{ Current Data Set and Charvet et al. (2013) Combined } \\
\hline$T$ & \multirow[t]{5}{*}{$R / a^{+}$} & 108.5 & Yes & 145.6 & 0.75 \\
\hline$\lambda$ & & 267.6 & Yes & 148.1 & 0.74 \\
\hline$\xi$ & & 1.278 and 2.972 & Yes & 156.9 & 0.73 \\
\hline$\lambda \sin (\beta) / d$ & & 79.05 & Yes & 141.1 & 0.77 \\
\hline$\xi \lambda \sin (\beta) / d$ & & 223.9 & Yes & 141.7 & 0.77 \\
\hline
\end{tabular}

$$
\frac{R}{a^{+}}=-0.0059\left(\frac{\lambda \sin (\beta)}{d}\right)+2.146, \quad \text { for } \quad \frac{\lambda \sin (\beta)}{d} \geq 79
$$

\subsection{Discussion}

The analysis in $\S 4.2$ shows that the breakpoint at which the transition between $R>a^{+}$ and $R \approx a^{+}$appears to be dependent on $\lambda \sin (\beta) / d$. This accounts for the apparent lack of shoaling in long waves by including information on the slope and the wavelength. It is not physically convincing that there is a defined breakpoint between shoaling and non-shoaling long waves. It is more reasonable that this breakpoint is diffuse, and depends on the values of $d$ and $\beta$. If verified, the relative slope length might be used to predict runup for a given wavelength and amplitude at a given depth of definition over a given slope. However, due to its empirical nature, recourse to expanded data sets that vary $\beta$ and $d$ is required to gain confidence in the ability of relative slope length to predict $R / a^{+}$, as well as define the physical reasonableness of a defined breakpoint. This is the aim of ongoing numerical modelling work.

The presented data set poses an interesting question regarding the definition of amplitude. While the shorter waves shoal the longer non-shoaling waves are effectively 'pre-shoaled' in the water depth that they are generated. In greater water depths, these waves would be generated with smaller amplitudes, corresponding to a longer slope over which they will propagate. In the latter cases the value $R / a^{+}$would proportionately increase, implying that 
normalising with $d$, while appropriate for solitary waves (due to the tie between depth and non-linearity), is not appropriate for tsunami-length trough or crest-led waves. Normalising these waveforms with depth appears arbitrary, which suggests that the depth at which the amplitude of the wave is defined may have significant consequences on the final prediction of the runup. The Iribarren number is used in classical wind wave runup equations with an assumption that deep water conditions apply. Theoretically, a tsunami wave will always feel the ocean bottom throughout its propagation, violating this assumption. This influenced the use of the relative slope length parameter to describe their runup.

These observations have implications for numerical studies of runup and may require consideration in guidance provided for how, and at what offshore depth tsunami waves should be defined. For example, the ASCE/SEI (2017) standard states that the offshore driving boundary condition for an $\mathrm{N}$-wave tsunami waveform is defined at a contour depth of $100 \mathrm{~m}$ (Eq. 6.7.1-1, therein). This depth contour is also used in Park et al. (2015) as a reasonable offshore depth to define a crest-led tsunami waveform prior to wave breaking closer to the shore, and far enough from the source to account for refractive and shoaling effects. The assumption is of less uncertainty in the tsunami propagation from source to the $100 \mathrm{~m}$ contour. In the ASCE/SEI (2017) standard the propagation from the source to the $100 \mathrm{~m}$ depth contour is permitted to be made using linear shallow water wave equations. Thereafter towards the shore the wave is propagated using non-linear shallow water wave equations or equivalent modelling techniques to account for non-linear effects applicable to the specific prototype being considered. The findings of the current PLWG tests show the depth and distance from the reflecting region (in this case the sloping bathymetry) has important effects on the waveform at any given $X$ position. The presence of destructive or constructive interference from the reflected trough or crest may require consideration depending on the distance from the shore and wave celerity and wavelength. In Figure 5a-c the destructive effects of the natural trough reflection are observed in the free-surface closer to the PLWG. This has important implications for the input boundary condition amplitude for any runup prediction and could lead to undesirable underestimations of runup for a given tsunami wave if the input amplitude is lowered by the reflected trough. Equally overestimates of $R$ can result if the input amplitude fails to take into account the reflected trough.

This leads to the potentially problematic identification of a requisite baseline waveform in modelling tsunami and their runup. The issue is whether the wave as defined at a given depth and distance offshore is completely composed of the input wave only. The extremely 
long length and period of these waves means that reflections may come into play in an offshore definition scenario.

These questions and the verification of relative slope length as a suitable prediction tool for $R / a^{+}$are suggested for future research. This may include a numerical model to expand the current experimental data set to include variations in $d$ and $\beta$, as well as efforts to propose a baseline waveform. Such a baseline may go some way into dealing with the uncertainties described above.

\section{Conclusions}

Using a Pneumatic Long-Wave Generator (PLWG), an extensive set of trough and crest-led waves are generated with periods varying from $10 \mathrm{~s}$ to $230 \mathrm{~s}$ at model scale. It is shown that the PLWG can produce tsunami-length waves that are much longer than the $100 \mathrm{~m}$ long flume. These waves are stable along the sloping bathymetry and scalable to prototype tsunami length, amplitudes and water depths. Flow velocity profiles show well developed logarithmic profiles near the PLWG and at the bathymetry toe.

The runup of trough and crest-led waves of periods $\approx 100-230 \mathrm{~s}$ is approximately equivalent to the offshore amplitude. This is postulated to be due to insignificant shoaling resulting in these very long waves behaving similarly to a slosh. Waves of periods of less than $\approx 100 \mathrm{~s}$ did shoal, presenting runup greater than offshore amplitude. Existing runup equations, with the exception of (Madsen and Schäffer, 2010) perform poorly for tsunamilength waves, in one case over-estimating by a factor of up to approximately 5 . Large under predictions are observed for tsunami length elevated waves. The equation provided by (Madsen and Schäffer, 2010) gave better results, but was unable to match the whole data set. The correlation of wave variables with runup is investigated and wave steepness is found to be strongly correlated with runup, indicating the distribution of energy over the waveform appears more important than the total value of potential energy. This energy distribution is better described by geometric variables, particularly the wave steepness measure. Using a segmented regression, a new parameter called the 'Relative Slope Length' is found to fit the data well. This includes information on the wavelength of the wave and the slope over which it travels.

The discussion and analysis of the long wave data set presented implies the depth at which a tsunami wave is defined is a key variable in determining whether its amplitude is absolute (the actual amplitude of the generated incident tsunami) or relative (the amplitude recorded 
in a particular position, possibly altered by wave interference). Reflected components of the incident wave are shown to interfere with the rear portions of the wave. For trough-led waves the crest amplitude may be decreased by the reflected trough. This suggests that runup models need to take into account the wavelength, celerity and depth at which the tsunami wave is defined to consider the effect of reflections on the amplitude and its definition.

The tests show that the definition of offshore wave amplitude is non-trivial and may greatly affect the predicted relative runup of a given wave. This appears to be a general issue for all types of tsunami simulation in the laboratory. Together these observations and proposed runup model provide a framework for future numerical studies of the topic.

\section{Acknowledgements}

This work is fully funded by the European Research Council project "URBANWAVES" [Starting Grant: 336084]. The experiments use the 2nd generation Tsunami Generator developed and constructed by HR Wallingford and operated onsite at HR Wallingford. Particular thanks is reserved for Dr Ioanna Ioannou, Department of Civil, Environmental and Geomatic Engineering, University College London, who performed the regressions for the empirical functions. The input of other researchers to these experiments is gratefully acknowledged. In no specific order: Dr Andrew Foster, Dr Crescenzo Petrone, Prof Ian Eames and Mr Oliver Cook from University College London, Mr Ignacio Barranco Granged from the National University of Singapore and Dr Ingrid Charvet of Risk Management Solutions.

\section{References}

J. Brown, Waves, Tides and Shallow Water Processes, Elsevier, 2013.

J. Telford, J. Cosgrave, R. Houghton, Synthesis Report: Joint evaluation of the international response to the Indian Ocean tsunami, Tech. Rep., Tsunami Evaluation Coaliton (TEC), 2006.

NOAA, Natural Hazards Viewer. National Centers for Environmental Information. National Oceanic and Atmopsheric Adminstration., URL https://maps.ngdc.noaa.gov/viewers/hazards, accessed 18/07/17, 2017a.

NOAA, Recent Significant Tsunami Events, URL https://ngdc .noaa.gov/hazard/recenttsunamis-tab accessed 18/07/17, 2017b. 
E. L. Geist, T. Parsons, Assessing historical rate changes in global tsunami occurrence, Geophysical Journal International 187 (2011) 497-509.

NPA, Damage Situation and Plice Countermeasures associated with the 2011 Tohoku district - off the Pacific Ocean Earthquake December 9, 2011, 2016.

Y. Kajitani, S. E. Chang, H. Tatano, Economic Impacts of the 2011 Tohoku-Oki Earthquake and Tsunami, Earthquake Spectra 29 (S1) (2013) S457-S478.

C. E. Synolakis, The runup of solitary waves, Journal of Fluid Mechanics 185 (1987) 523-545.

S. Tadepalli, C. Synolakis, The Runup of N-waves, Proceedings of the Royal Society A 445 (1994) 99-112.

M. J. Briggs, C. E. Synolakis, G. S. Harkins, D. R. Green, Laboratory Experiments of Tsunami Runup on a Circular Island, Journal of Pure and Applied Geophysics 144 (3) (1995) 569-593.

P. L.-F. Liu, Y.-S. Cho, M. J. Briggs, U. Kanoglu, C. E. Synolakis, Runup of Solitary Waves on a Circular Island, Journal of Fluid Mechanics 302 (1995) 259-285.

S. A. Hughes, Estimation of Wave Run-up on Smooth, Impermeable Slopes Using the Wave Momentum Flux Parameter, Coastal Engineering 51 (2004a) 321-332.

P. A. Madsen, H. Schäffer, Analytical Solutions for Tsunami Runup on a Plane Beach: Single Waves, N-waves and Transient Waves, The Journal of Fluid Mechanics 645 (2010) 27-57.

I. Charvet, I. Eames, T. Rossetto, New tsunami runup relationships based on long wave experiments., Ocean Modelling 69 (2013) 79-92.

G. Saelevik, A. Jensen, G. Pedersen, Runup of Solitary Waves on a Straight and a Composite Beach, Coastal Engineering 77 (2013) 40-48.

V. Sriram, I. Didenkulova, A. Sergeeva, S. Schimmels, Tsunami Evolution and Run-up in a Large Scale Experimental Facility, Coastal Engineering 111 (2016) 1-12.

U. Drähne, N. Goseberg, S. Vater, N. Beisiegel, J. Behrens, An Experimental and Numerical Study of Long Wave Run-Up on a Plane Beach, Journal of Marine Science and Engineering $4(1)$. 
F. Imamura, Tsunamis: Ideas and Observations on Progress in the Study of the Seas, chap. 10 - Tsunami Modeling: Calculating Inundation and Hazard Maps, Harvard University Press, 321-332, 2009.

ASCE/SEI, Chapter 6 Tsunami Loads and Effects, ASCE/SEI 7-16 .

H. Taubenböck, N. Goseberg, G. Lämmel, N. Setiadi, T. Schlurmann, K. Nagel, F. Siegert, J. Birkmann, K. P. Traub, S. Dech, V. Keuck, F. Lehmann, G. Strunz, H. Klüpfel, Risk reduction at the "Last-Mile": an attempt to turn science into action by the example of Padang, Indonesia, Natural Hazards 65 (2013) 915-945, doi:10.1007/s11069-012-0377-0.

Y.-H. Chang, K. S. Hwang, H.-H. Hwung, Large-scale Laboratory Measurements of Solitary Wave Inundation on a 1:20 Slope, Coastal Engineering 56 (10) (2009) 1022-1034.

P. Madsen, D. Fuhrman, H. Schäffer, On the Solitary Wave Paradigm for Tsunamis, Journal of Geophysical Research 113 (C12012).

N. Goseberg, A. Wurpts, T. Schlurmann, Laboratory-scale Generation of Tsunami and Long Waves., Coastal Engineering 79 (2013) 57-74.

G. C. Bremm, N. Goseberg, T. Schlurman, I. Nistor, Long Wave Flow Interaction with a Single Square Structure on a Sloping Beach, Journal of Marine Science and Engineering 3 (2015) 821-844.

S. Schimmels, V. Sriram, I. Didenkulova, Tsunami Generation in a Large Scale Experimental Facility, Coastal Engineering 110 (2016) 32-41.

H. Fernández, V. Sriram, S. Schimmels, H. Oumeraci, Extreme Wave Generation Using Self Correcting Method - Revisited, Coastal Engineering 93 (2014) 15-31.

T. Rossetto, W. Allsop, I. Charvet, D. Robinson, Physical Modelling of Tsunami using a New Pneumatic Wave Generator., Coastal Engineering 58 (2011) 517-527.

W. Allsop, I. Chandler, M. Zaccaria, Improvements in the Physical Modelling of Tsunamis and their Effects, in: Proceeding of the 5th International Conference on Application of Physical Modelling to Port and Coastal Protection, Keynote paper to CoastLab 2014, 2014. 
I. Chandler, W. Allsop, I. Barranco, D. J. McGovern, Understanding Wave Generation in Pneumatic Tsunami Simulators, in: Proceedings of the 6th International Conference on the Application of Physical Modelling in Coastal and Port Engineering and Science (Coastlab16), 2016.

D. G. Goring, V. I. Nikora, Despiking Acoustic Doppler Velocimeter Data, Journal of Hydraulic Engineering 128 (2002) 117-126.

S. T. Grilli, J. C. Harris, T. S. Tajalli Bakhsh, T. L. Masterlark, C. Kyriakopoulos, J. T. Kirby, F. Shi, Numerical Simulation of the 2011 Tohoku Tsunami Based on a New Transient FEM Co-seismic Source: comparison to Far- and Near-field Observations, Pure and Applied Geophysics (170) (2013) 1333-1359.

T. S. Hedges, Regions of validity of analytical wave theories., Proceedings of the Institute of Civil Engineering - Water and Maritime Engineering 112 (1995) 111-114.

S. A. Hughes, Physical Models and Laboratory Techniques in Coastal Engineering, Advanced Series on Ocean Engineering, World Scientific Publishing Co, 1995.

J. Peakall, J. Warburton, Surface tension in small hydraulic river models-The significance of the Weber number., Journal of Hydrology 53 (1996) 199-212.

V. Heller, Scale Effects in Physical Hydraulic Engineering Models, Journal of Hydraulic Research 49 (3) (2011) 293-306.

D. J. McGovern, I. D. Chandler, T. Rossetto, Experimental Study of the Runup of Tsunami Waves on a Smooth Sloping Beach, in: Proceedings of the 6th International Conference on the Application of Physical Modelling in Coastal and Port Engineering and Science (Coastlab16), 2016.

J. J. V. Hall, G. M. Watts, Laboratory investigation of the vertical rise of solitary waves on impermeable slopes, Tech. Rep. 33, Beach Erosion Board, U.S. Army Corps of Engineers, 1953.

S. A. Hughes, Wave momentum flux parameter: a descriptor for nearshore waves, Coastal Engineering 51 (2004b) 1067-1084. 
J. Battjes, Surf Similarity, in: Coastal Engineering Proceedings: Proceedings of 14th Conference on Coastal Engineering, Copenhagen, Denmark, 14, 1974.

H. Mase, Random Wave Runup Height on Gentle Slope, Journal of Waterway, Port, Coastal, and Ocean Engineering, 115 (5) (1989) 649-661.

M. A. Losada, L. A. Giménez-Curto, Flow Characteristics on Rough, Permeable Slopes Under Wave Action, Coastal Engineering 1 (1981) 187-206.

N. Goseberg, Reduction of maximum tsunami run-up due to the interaction with beachfront development - application of single sinusoidal waves, Natural Hazards and Earth System Sciences 13 (2013) 2991-3010.

D. V. Hinkley, Inference in Two-Phase Regression, Journal of the American Statistical Association 66 (336) (1971) 736-743.

H. Akaike, A new look at the statistical model identification, doi:10.1109/TAC.1974.1100705, URL http: //ieeexplore.ieee.org/document/1100705/?, 1974.

V. M. Muggeo, segmented: an R Package to Fit Regression Models with Broken-Line Relationships., R News 8 (1) (2008) 20-25, URL https ://cran.r-project.org/doc/Rnews/.

H. Park, D. T. Cox, C. M. Petroff, An Empirical Solution for Tsunami Run-up on Compound Slopes, Natural Hazards 76 (2015) 1727-1743.

A. B. Rabinovich, R. E. Thomson, The 26 December 2004 Sumatra tsunami: analysis of tide gauge data from the World Ocean Part 1. Indian Ocean and South Africa., Pure and Applied Geophysics (164) (2007) 261-308.

H. Kawai, M. Satoh, K. Kawaguchi, K. Seki, Characteristics of the 2011 Tohoku tsunami waveform acquired around Japan by NOWPHAS equipment., Coastal Engineering Journal (55: 1350008-1-27).

\section{Appendix A.}

Example prototype tsunami time series showing variable trough-led and crest-led type waveforms from a) the Belgian Yacht 'Mercator' recorded during the Indian Ocean Tsunami 
a)

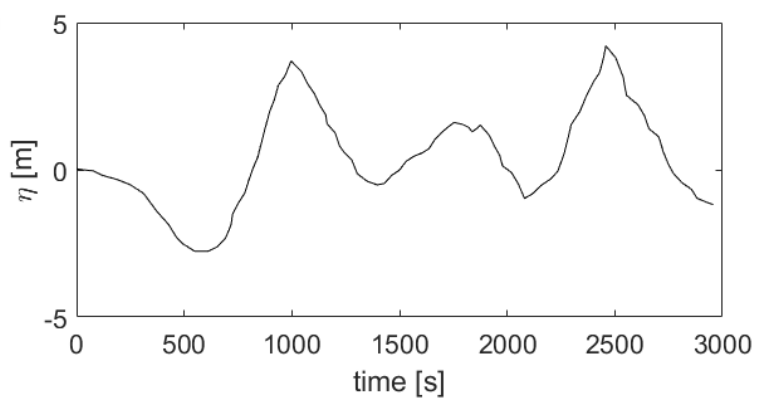

c)

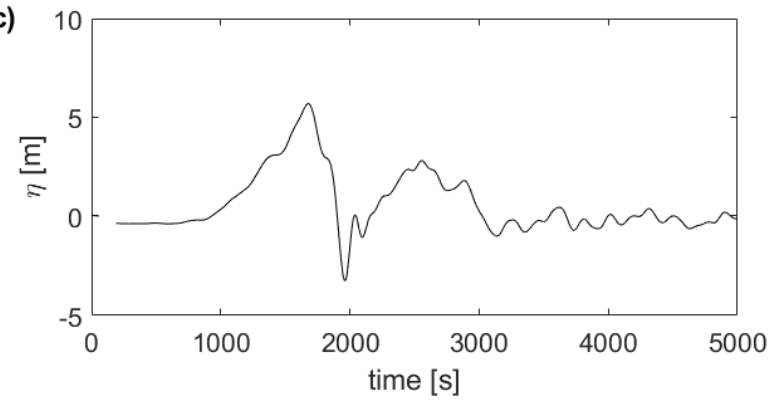

b)

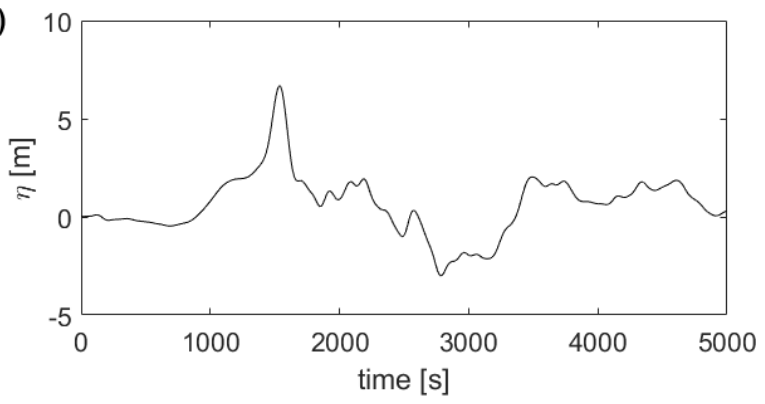

d)

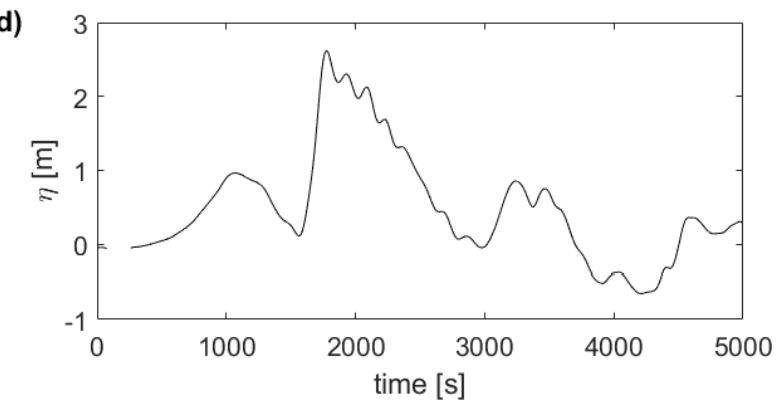

Figure A.1: prototype tsunami time series examples from a) 'Mercator' yacht, b) Iwate South, c) Miyagi North, and d) Fukushima tide gauges

\section{Appendix B.}

Table B.1 gives the test conditions and the standard deviations of the mean of repeated tests, where appropriate. 


\begin{tabular}{|c|c|c|}
\hline 0 & & 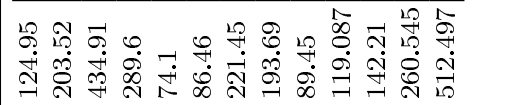 \\
\hline$x^{2}$ & $\frac{g}{g}$ & 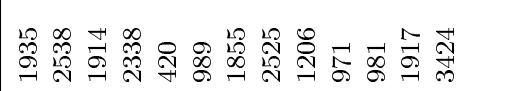 \\
\hline 0 & & 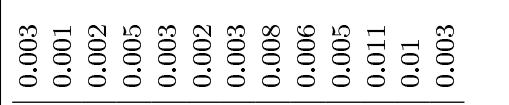 \\
\hline$\infty$ & $\bar{g}$ & 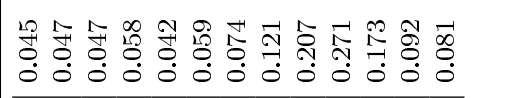 \\
\hline 6 & & 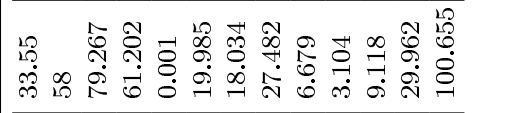 \\
\hline r & $\bar{g}$ & 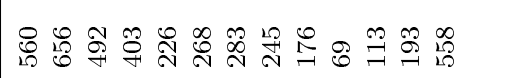 \\
\hline 6 & & 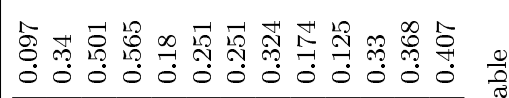 \\
\hline o & $\begin{array}{l}F \\
\bar{F} \\
F_{0} \\
g\end{array}$ & 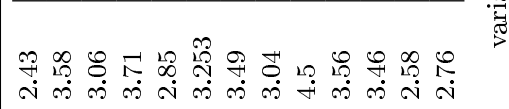 \\
\hline 6 & & 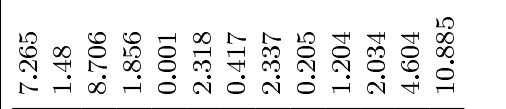 \\
\hline$H$ & $\bar{g}$ & 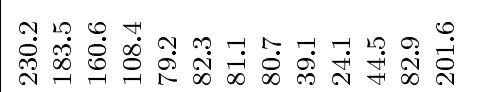 \\
\hline ○ & & 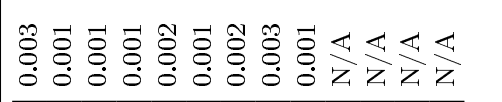 \\
\hline 'o & $\underline{\underline{g}}$ & 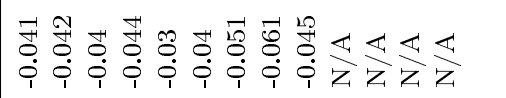 \\
\hline 0 & & 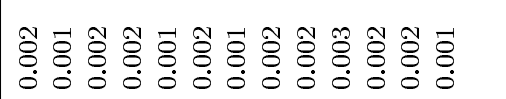 \\
\hline$t_{*}^{+}$ & $\bar{g}$ & 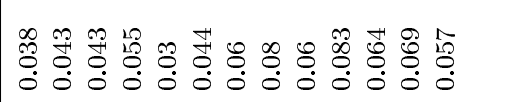 \\
\hline 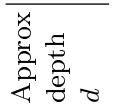 & $\bar{g}$ & - \\
\hline 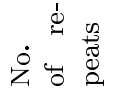 & & 100010101090 \\
\hline 宽: & $\underline{g}$ & 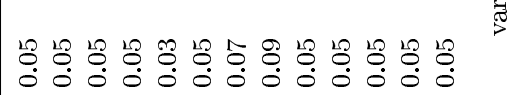 \\
\hline 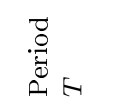 & 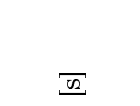 & 品 \\
\hline$\sum_{\substack{0 \\
\vdots}}^{\infty}$ & 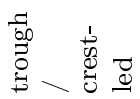 & 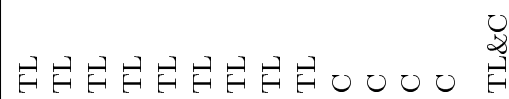 \\
\hline
\end{tabular}




\section{Appendix C.}

In $\S 4.2$ a segmented regression analysis is given. In determining which influencing variables may depict $R / a^{+}$, the following correlation plots with the combined data sets (current and Charvet et al. 2013), including the aggregated repeat waves, is given in Figure C.1. The CDF plot in Figure 14, alongside the quantile-quantile and probability-probability plots in Figure C.2, show the normal distribution fits the $R / a^{+}$data better than the $\log$ normal or gamma distribution counterparts. Correlations are apparent in $\lambda \sin (\beta) / d, \xi / d$, $\xi(\lambda \sin (\beta) / d), T, E_{p}$ and $\lambda$.
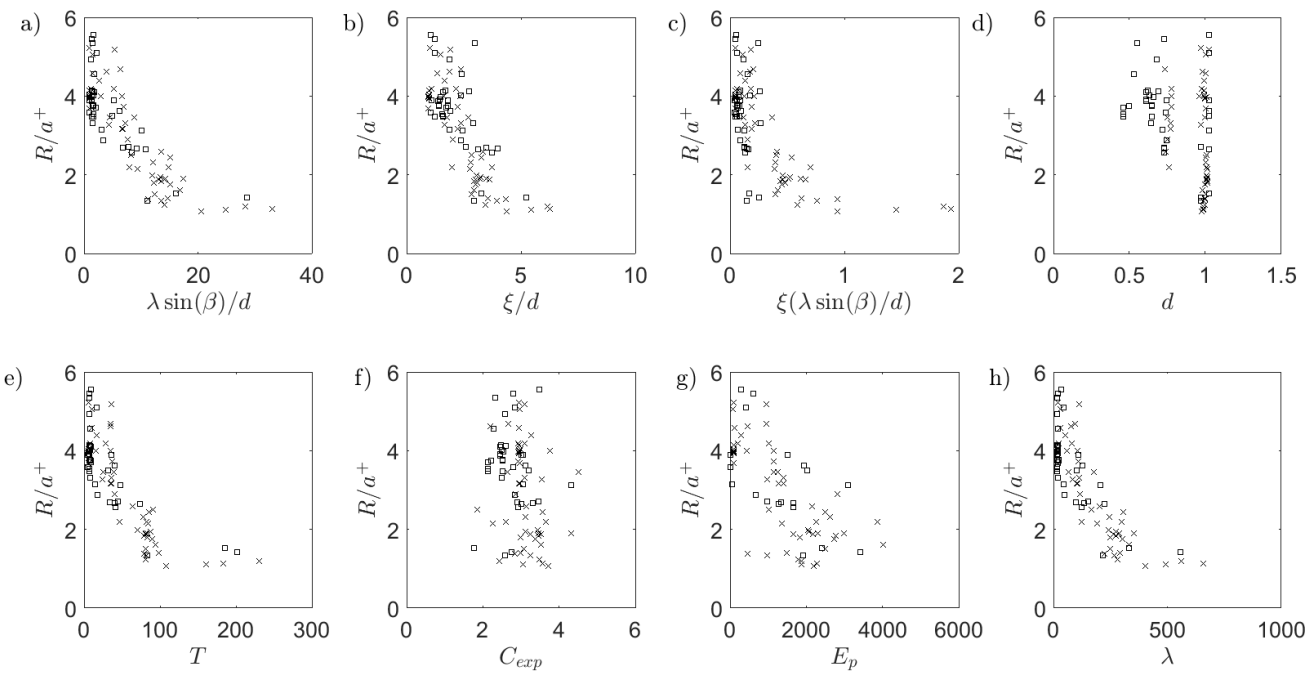

Figure C.1: Correlation plots of all potentially influencing variables as a function of $R / a^{+}$tested in the combined data sets of the current data and Charvet et al. (2013).

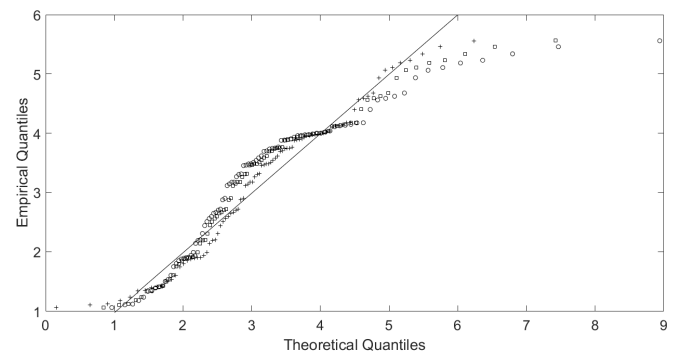

(a) Quantile-quantile

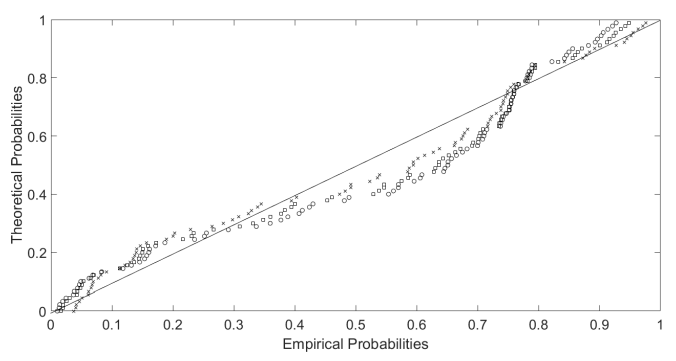

(b) Probability-probability

Figure C.2: Quantile-quantile (a) and probability-probability (b) comparison plots of the normal $(\times)$, lognormal (o) and gamma ( $\square$ ) cumulative distribution function (CDF) fits to $R / a^{+}$ 